Hae Jin Choi

Seung Jae Lee

Hyo Jae Jo

Gang Nam Lee

Kwang Hyo Jung

http://dx.doi.org/10.21278/brod69102

ISSN 0007-215X

eISSN 1845-5859

\title{
COMPARISON STUDY OF EXPERIMENTS AND PREDICTIONS OF WAVE KINEMATICS FOR ROGUE WAVE
}

\author{
UDC 629.5.016.8: 629.5.017.2: 629.5.018.1 \\ Original scientific paper
}

\begin{abstract}
Summary
To investigate the wave kinematics under the rogue wave crest, a series of experiments were performed in 2-D wave tank with the application of PIV technique to measure the velocities under the free surface. Three different prediction methods of linear extrapolation, Wheeler stretching, and modified stretching were applied to estimate water wave kinematics and compared with PIV experimental results under the highest wave crest of irregular wave trains satisfying with rogue wave criteria. Also, the cut-off frequency dependence for three prediction methods was investigated with varying spectral peak frequencies to estimate wave kinematics including velocities and accelerations in horizontal and vertical directions. It was suggested that the cut-off frequency for the reasonable prediction of the wave kinematics under the rogue wave crest could be chosen three times of spectral peak wave frequency for the linear extrapolation and higher frequency than four times of spectral peak wave frequency for Wheeler stretching and modified stretching method.
\end{abstract}

Key words: $\quad$ rogue wave; wave kinematics; cut-off frequency; linear extrapolation; Wheeler stretching; modified stretching

\section{Introduction}

For the last several decades, the damages of many ships and offshore structures have been caused of rogue waves which had an exceptional height and abnormal shape. Two large Norwegian bulk ships M/S "Norse Variant" and M/S "Anita" disappeared at the same time at the same location [1]. According to the conclusion of the Court of Inquiry, a very large wave suddenly broke several hatch covers on deck, and the ships were filled with water and sank before any emergency call was given. The Queen Elizabeth II was struck by a rogue wave of 29 m wave height in 1995 in the North Atlantic [2]. The Caledonian Star, sailing in the South Atlantic in 2001, was hit by a rogue wave estimated to be $30 \mathrm{~m}$, approximately, of wave height. The Explorer, on a "semester-at-sea" sailing in the North Pacific, was damaged in January 2005 when the ship, carrying nearly 1000 people including almost 700 college students, was struck by a wave estimated to be $17 \mathrm{~m}$ in height. The wall of water smashed into the bridge of the 180 $\mathrm{m}$ long ship. These well-built cruise ships suffered a little damage and had a few injuries from 
the attack of rogue waves [3]. The Norwegian Dawn, a 3 years old $294 \mathrm{~m}$ long cruise ship carrying more than 2200 passengers and heading back to New York from the Bahamas, was pounded by a rogue wave during a storm in April 2005 off the South Carolina coast. The wave reached the $10^{\text {th }}$ deck of the towering ship, and 62 cabins were flooded and some public areas were damaged [4]. Offshore platform "Draupner oil rig" in the North Sea have also been meet with the rogue wave of $26 \mathrm{~m}$ in height measured by an onboard laser, while the surrounding waves reached $12 \mathrm{~m}$ on January 1, 1995 [5]. MaxWave, a German scientific group, examined 30,000 worldwide satellite photos taken by the European Space Agency (ESA). According to MaxWave, 10 rogue waves, each larger than $25 \mathrm{~m}$ in height, were identified around the globe within the three-week research period in 2001 [6]. These rogue waves occur more frequently where there are strong currents, such as the Gulf Stream off the eastern coast of North America.

After Draper [7] suggested using the term "freak waves" and developed a theory for application to a real ocean wave spectrum, the terms for waves having exceptional high wave height varied as extreme waves, giant waves, mountain waves, rogue waves, etc. Recently, many researchers have used "rogue waves" as academic terms. Researchers $[8,9]$ defined rogue wave as a wave height exceeding twice of the significant wave height of surrounding waves. Olagnon and Iseghem [10] defined a rogue wave when the wave height was larger than two times of significant wave height and the ratio of crest height to significant wave height was larger than 1.25 .

The factors of wind-driven waves, currents, ocean bottom topography or inclement weather can play a role in rogue wave development. With the assumption of the linear wave theory, rogue waves can be considered as the superposition of a number of independent monochromatic waves with different frequencies and directions. A rogue wave may appear in the process of geometrical focusing, dispersion enhancement, and wave-current interactions. Kharif et al. [11], and Smith and Swan [12] explained the rogue wave phenomenon with an aspect of dispersion enhancement in a random sea. The combination of the geometrical focusing and dispersion enhancement mechanism to form an extreme wave has also been examined by Wu and Nepf [13]. Peregrine [14] and Smith [15] investigated the rogue waves with the mechanism of wave-current interactions for the Agulhas current in the South Africa where strong currents are often observed. Levrenov [16] described the rogue waves in Agulhas with an aspect on the concentration of wave-energy density.

Three processes mentioned above are investigated analytically and numerically in the framework of weakly nonlinear models like the nonlinear Schrödinger equation, as well as researched in the laboratory. Tulin and Waseda [17] observed highly nonlinear waves including breaking waves in deep water in a large wave tank. Trulsen and Dysthe $[18,19]$ focused on the numerical simulation of rogue waves with 3rd and 4th order nonlinear Schrödinger equations and provided breather type solutions using the equations suggested by Henderson et al. [20]. Osborne et al. [21, 22] studied the dynamic behaviour of rogue waves with the numerical model and extended to the directional, random oceanic sea states. Lu et al. [23] adopted the wavelet analysis method to analyse the rogue waves to the time-frequency energy distribution. Zhang and Soares [24] investigated the ship responses to rogue waves simulated by the nonlinear Schrödinger equation.

Nonlinear wave-wave interaction has been addressed in association with the rogue wave formation [25, 26]. Grue [27], and Clamond and Grue [28] performed a fully nonlinear numerical simulation of a long time evolution using a two-dimensional localized long wave packet. In a 2-D wave tank, Wu and Yao [29] investigated rogue wave kinematics using a combined mechanism of dispersion enhancement and wave-current interaction. Zou and Kim [30] generated a strongly asymmetric wave in the 2-D wave tank by the time series distortion to the highest wave in irregular wave train. Kim and Kim [31] simulated the Draupner rogue 
wave in the 2-D wave tank with measuring the horizontal particle velocity and the horizontal force on a vertical truncated cylinder fixed in the wave. In the towing tank, Bennett et al. [32] provided a technique for experimental modelling of rogue waves using NewWave method.

In this study, PIV (particle image velocimetry) technique was employed to measure a water particle velocity profile under the wave crest, and measured water velocity components was verified by comparing with theoretical results of regular waves. The rogue wave was generated with the distortion method applying to the highest wave in irregular wave train of JONSWAP spectrum in 2-D wave tank. The water velocity profiles under rogue wave crests were measured by PIV technique, and their local and convective accelerations were calculated from consecutive and individual velocity profiles, respectively. Water kinematics of rogue waves were compared with results with three prediction methods of the linear extrapolation, Wheeler stretching [33], and the modified stretching [34], and their cut-off frequency dependences were investigated on the wave kinematics prediction under wave crests of rogue waves.

\section{Experimental setup and technique}

A series of experiments was conducted to measure the water particle velocities of regular and irregular water waves in 2-D wave tank which is $35 \mathrm{~m}$ long, $0.91 \mathrm{~m}$ wide, and $1.22 \mathrm{~m}$ deep glass-walled flume as shown in Fig 1. It was equipped with a permeable wave absorbing 1:5.5 sloped beach having 5\% reflection. Wave maker had a dry-back, hinged flap type and was driven by a synchronous servo-motor controlled by a computer and hydrostatically balanced using an automatic near constant force and a pneumatic control system. The set-up is shown schematically in Fig. 1, where $\mathrm{x}$ is the horizontal coordinate positive in the direction of wave propagation from the wave maker and $\mathrm{z}$ is positive upward. Free surface elevations of regular and irregular waves were measured at $800 \mathrm{~cm}$ from the wave maker, respectively, and the water depth in the wave tank was maintained at $90 \mathrm{~cm}$.

Regular waves having the wave period of $0.9 \mathrm{~s}$ were generated with three different wave heights (Table 1), and four irregular wave trains using JONSWAP spectrum with a peak enhancement factor of 6.5 were generated in 2-D wave tank (Table 2). The distortion method [33] was applied at the highest wave crest in order to increase the maximum height of irregular wave trains. The maximum wave heights of in irregular waves of cases PH3 and PH4 were satisfied with the rogue wave criteria listed in Table 2.

Table 1 Wave parameters of regular waves ( $H_{C}$ : Wave crest height, $H_{t}$ : Wave trough height)

\begin{tabular}{|c|c|c|c|c|c|c|}
\hline Case & $\boldsymbol{H}(\mathbf{c m})$ & $\boldsymbol{H}_{\boldsymbol{C}}(\mathbf{c m})$ & $\boldsymbol{H}_{\boldsymbol{t}}(\mathbf{c m})$ & $\boldsymbol{T}(\mathbf{s})$ & $\boldsymbol{H} / \boldsymbol{L}$ & $\boldsymbol{k} \boldsymbol{a}$ \\
\hline PR1 & 4.17 & 2.05 & -2.12 & 0.90 & 0.033 & 0.104 \\
\hline PR2 & 8.13 & 4.52 & -3.61 & 0.90 & 0.064 & 0.202 \\
\hline PR3 & 12.29 & 7.27 & -5.02 & 0.90 & 0.097 & 0.305 \\
\hline
\end{tabular}

Table 2 Wave parameters of irregular waves

\begin{tabular}{|c|c|c|c|c|c|}
\hline Case & $\boldsymbol{H}_{\boldsymbol{S}}(\mathbf{c m})$ & $\boldsymbol{T}_{\boldsymbol{S}}(\mathbf{s})$ & $\boldsymbol{H}_{M a x}(\mathbf{c m})$ & $\boldsymbol{H}_{M a x} / \boldsymbol{H}_{\boldsymbol{S}}$ & $\boldsymbol{H}_{\boldsymbol{C}} / \boldsymbol{H}_{\boldsymbol{S}}$ \\
\hline PH1 & 6.63 & 1.25 & 14.11 & 2.13 & 1.20 \\
\hline PH2 & 7.00 & 1.27 & 15.11 & 2.16 & 1.22 \\
\hline PH3 & 7.43 & 1.19 & 16.09 & 2.17 & 1.25 \\
\hline PH4 & 7.78 & 1.18 & 16.36 & 2.11 & 1.29 \\
\hline
\end{tabular}


The PIV technique was employed to obtain the velocity field of water waves. The PIV system and the wavemaker were synchronized by computer A housing a data acquisition board (Fig. 1). Computer B saved wave elevation from the wave gage, and the timing of laser pulses and CCD camera were controlled by computer $\mathrm{C}$ housing the Programmable-Timing-UnitBoard. The digital CCD camera mounted with a $105 \mathrm{~mm} \mathrm{f} / 1.8$ micro focal lens set at $\mathrm{f} / 2.8$ 4.0 was used to obtain PIV images. It had $1280 \times 1024$ pixels, $6.7 \mu \mathrm{m} \times 6.7 \mu \mathrm{m}$ pixel size, 12 bit dynamic range, and $8 \mathrm{~Hz}$ framing rate. A pair of images was obtained by the doubleframe/single-pulsed method shown in Fig. 2. The time difference $(d t)$ between the $1^{\text {st }}$ frame and the $2^{\text {nd }}$ frame was adjusted to be about $3 \sim 5 \mathrm{~ms}$, which was determined by the maximum displacement to be less than a third of the width of the interrogation window size. The fields of view (FOV) sizes were $127 \times 159 \mathrm{~mm}^{2}$ and $172 \times 215 \mathrm{~mm}^{2}$ for regular and irregular wave conditions, respectively, corresponding to spatial resolutions of $2.01 \mathrm{~mm}$ and $2.72 \mathrm{~mm}$ between every velocity vectors in the interrogation area of $32 \times 32$ pixels with $50 \%$ overlap. The adaptive multi-pass algorithm was applied to reduce faulty vectors. Because this method has shifted an interrogation area to the location where particles moved, the stronger crosscorrelation can be taken. Once the velocity vectors have been calculated in the interrogation area with 50\% overlap, spurious false vectors were eliminated by the median filter [36]. The left-over empty spaces were filled-up with interpolated vectors and smoothed by a simple $3 \times$ 3 smoothing filter to reduce noise.

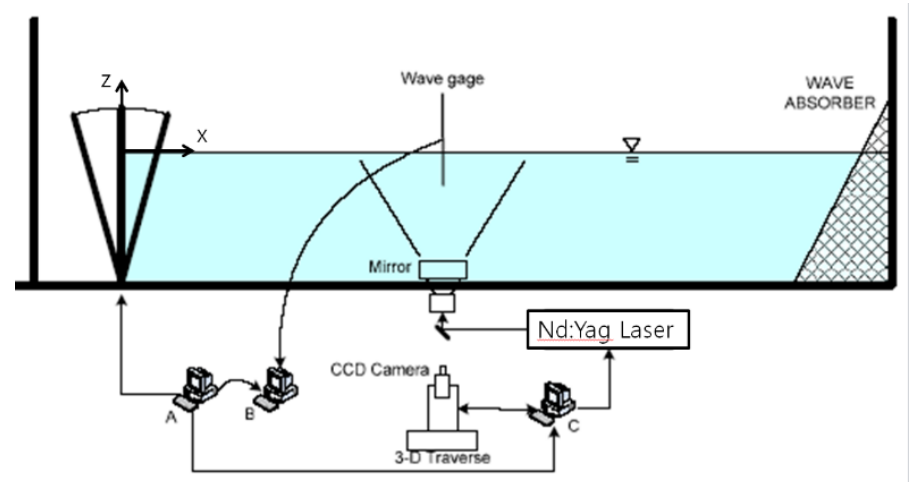

Fig. 1 Schematic sketch of the experimental set-up

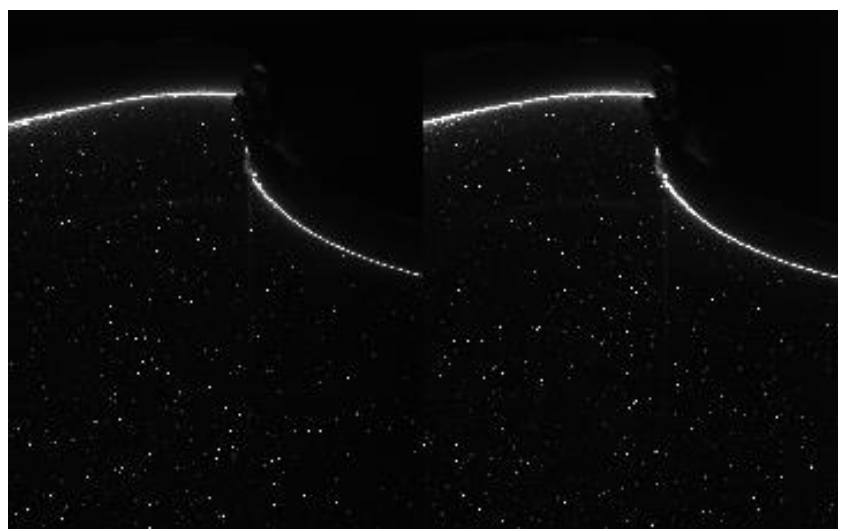

Fig. 2 Pair of images taken by the double-frame/single-pulsed method

The time series of wave elevation for the case PR2 was shown in Fig. 3, and the rootmean-square of wave heights $\left(H_{r m s}\right)$ were computed for each case of regular waves listed in Table 3. The RMS wave height was obtained by Equation (1), i.e. 
Comparison Study of Experiments and Predictions of Wave Kinematics for Rogue Wave
Hae Jin Choi, Seung Jae Lee, Hyo Jae Jo Gang Nam Lee, Kwang Hyo Jung

$$
H_{r m s}=\sqrt{\sum_{i=1}^{N}\left[H_{i}-H_{\text {mean }}\right)^{2} / N}
$$

where $H_{i}$ represents measured wave height for each wave period, $H_{\text {mean }}$ the average wave height of twelve selected regular wave trains, and $N$ the number of selected regular waves to be averaged. The error rate of selected regular waves was calculated by Equation (2).

$$
\mathrm{ER}_{\text {wave }}(\%)=\left(\frac{H_{r m s}}{H_{\text {mean }}}\right) \times 100
$$

The total error of the wave generation and the wave elevation measurement system was estimated below $1 \%$ for all regular wave conditions in Table 3.

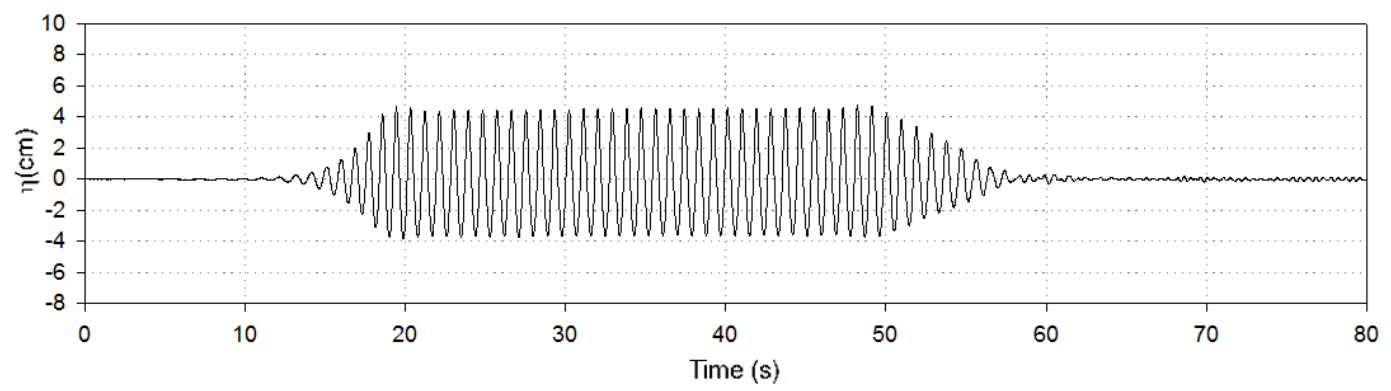

Fig. 3 Time series of regular waves for the case PR2, T= $0.9 \mathrm{~s}, \mathrm{H}=8.13 \mathrm{~cm}$

Table 3 RMS of wave heights from selected experimental regular waves

\begin{tabular}{|c|c|c|c|}
\hline Case & PR1 & PR2 & PR3 \\
\hline $\mathrm{H}_{\mathrm{rms}}(\mathrm{cm})$ & 0.01 & 0.02 & 0.07 \\
\hline $\mathrm{ER}_{\text {wave }}(\%)$ & 0.21 & 0.23 & 0.65 \\
\hline
\end{tabular}

To simulate the rogue wave in the 2-D wave tank, a series of irregular wave trains have been generated with JONSWAP spectrum (peak enhancement factor, $\gamma=6.5$ ). Two important characteristics of the rogue wave were considered for generating the rogue wave in the 2-D wave tank instead of in the ocean. The first characteristic was the wave height to be larger than twice of the significant wave height, and the second characteristics was the ratio of crest height $H_{c}$ to significant wave height $H_{s}$ to be greater than 1.25 [10]. The second characteristic of the rogue wave was presenting a strongly asymmetric wave profile which was one of typical patterns of highly nonlinear wave. 

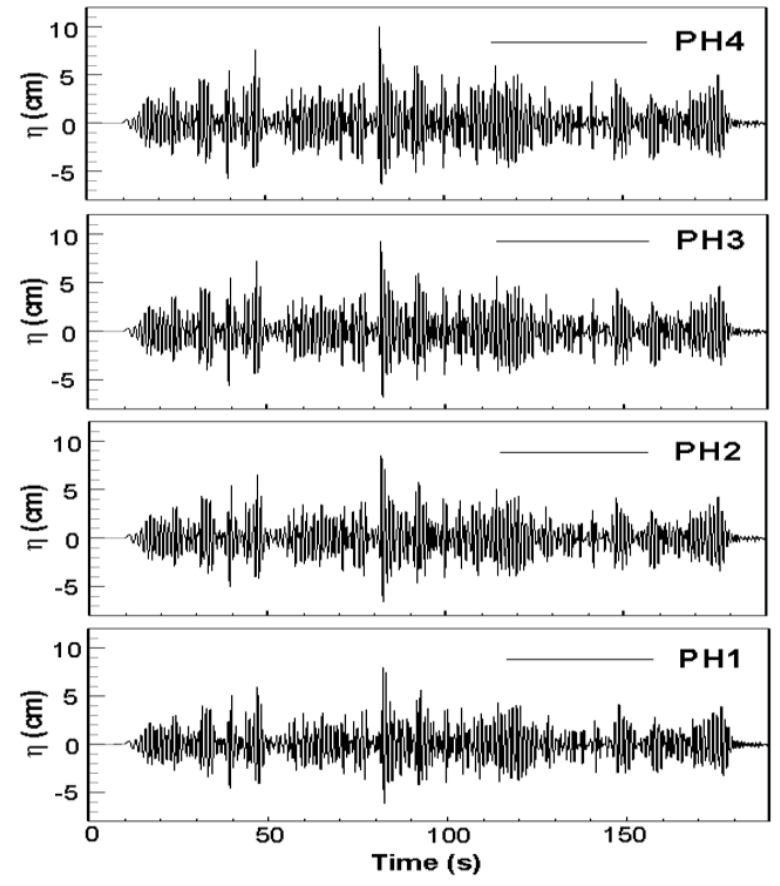

(a) Whole time series for four irregular waves

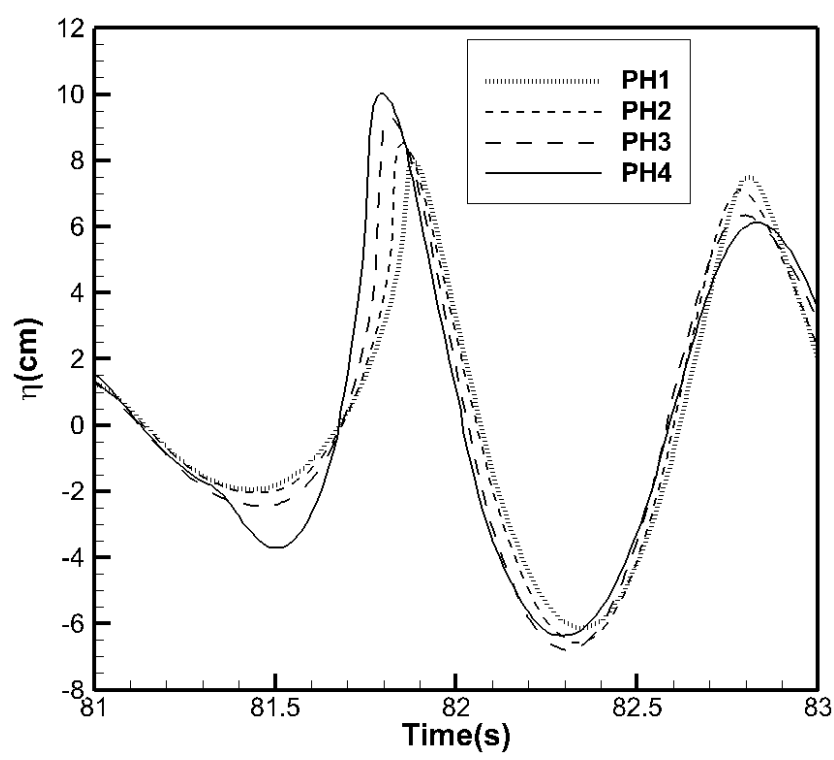

(b) Wave profiles at highest elevation

Fig. 4 Time series of four irregular waves

The distortion method [35] was applied to generate the rogue wave in 2-D wave tank, which has three steps of amplitude distortion, time distortion, and crest distortion techniques to increase the highest wave height and make a stronger asymmetry wave profile in the irregular wave. Amplitude distortion was intended to increase the crest height and reduce the trough height but kept the amplitude spectrum and changed the phase spectrum only. The time distortion made the duration of the trough to be longer and that of the crest to be shorter but its local wave period remained. Crest distortion was employed to move the location of the highest wave crest forward, and therefore, the front steepness would be increased. Fig. 4 showed the whole time series of four irregular wave trains and the comparison of strong asymmetric wave 
profiles. Wave profiles of the highest wave elevation in cases $\mathrm{PH} 3$ and $\mathrm{PH} 4$ met the rogue wave criteria. Amplitude spectra of cases $\mathrm{PH} 3$ and $\mathrm{PH} 4$ were presented with respect to the spectral peak frequency $\left(\omega_{p}=5.75 \mathrm{rad} / \mathrm{s}\right)$ in Fig. 5, which were going to be referred for the investigation of cut-off frequency dependence on the wave kinematics predictions.

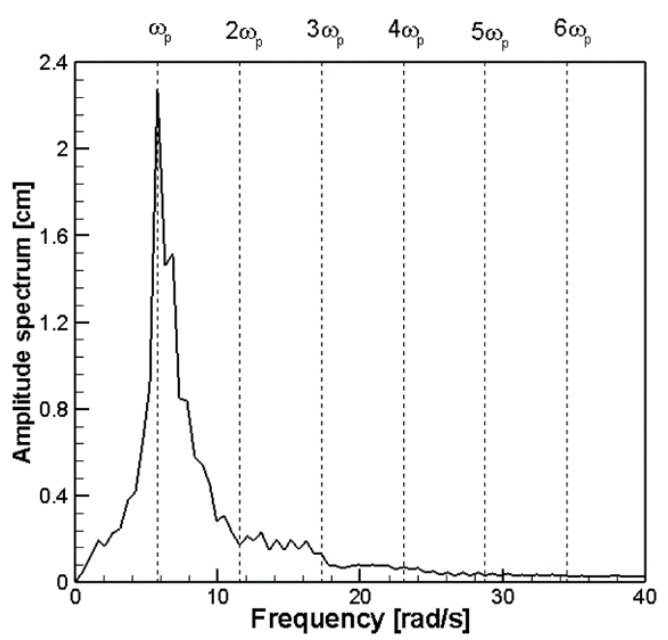

(a) Case PH3

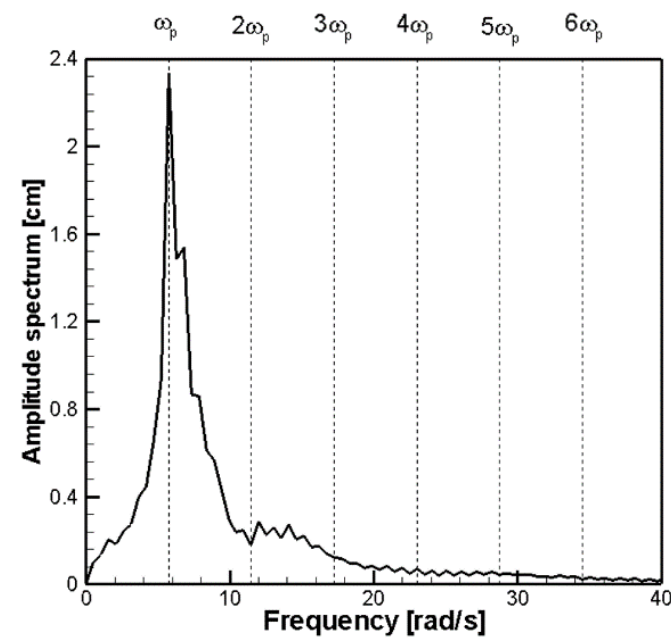

(b) Case PH4(left)

Fig. 5 JONSWAP spectra of rogue waves in 2-D wave tank $\left(\omega_{\mathrm{p}}=5.75 \mathrm{rad} / \mathrm{s}\right)$

To verify the applicability of PIV technique for the measurement of wave kinematics under the wave crest, PIV measurements were applied to obtain instantaneous velocity profiles for regular waves, and the mean velocity profiles were calculated with the phase-averaged method. Twelve pairs of images were taken at each phase in one wave length by PIV system. The mean velocity was obtained by phase-averaged method from twelve measured instantaneous velocities at each phase, i.e.,

$$
U_{k}=\frac{1}{N} \sum_{l=1}^{N} u_{k}^{(l)}
$$

where $U_{k}$ was the phase-averaged velocity, $u_{k}^{(l)}$ was the $k$-component velocity obtained from the $l$ th instantaneous velocity measurement, and $\mathrm{N}$ the total number of instantaneous velocities at that phase.

The mean velocity profiles were compared with results of the $3^{\text {rd }}$ order Stokes wave theory for regular waves of three wave heights. Horizontal velocity profiles for three regular waves were averaged from 12 instantaneous velocity profiles taken at the same phase of each wave length and were compared with those of the $3^{\text {rd }}$ order Stokes wave theory under the wave crest shown in Fig. 6. Averaged horizontal velocity profiles normalized by wave phase velocity $\left(V_{p}\right)$ were plotted with the respect to the vertical axis normalized by water depth $\mathrm{d}$. The experimental results showed the exponentially increasing horizontal water velocity up to the wave crest and agreed well with results of the $3^{\text {rd }}$ order Stokes wave theory for all three regular waves. 


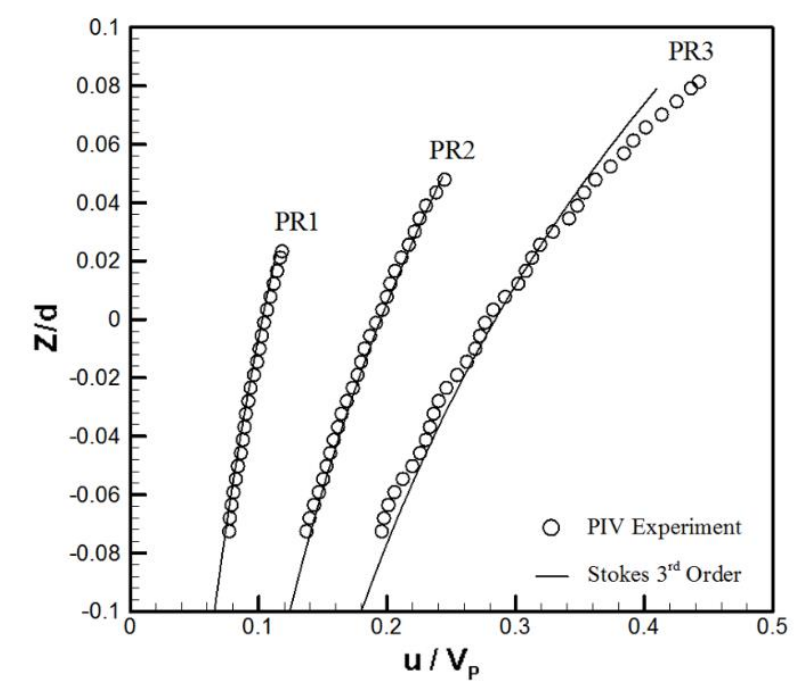

Fig. 6 Horizontal velocities under the wave crest of three regular waves

The RMS horizontal particle velocity was obtained by Equation (4), i.e.

$$
u_{r m s}=\sqrt{\sum_{i=1}^{N}\left(U-u_{i}\right)^{2} / N}
$$

where $U$ and $u_{i}$ were the phase-averaged velocity and the instantaneous measured velocity, respectively, and $N$ the number of selected regular waves.

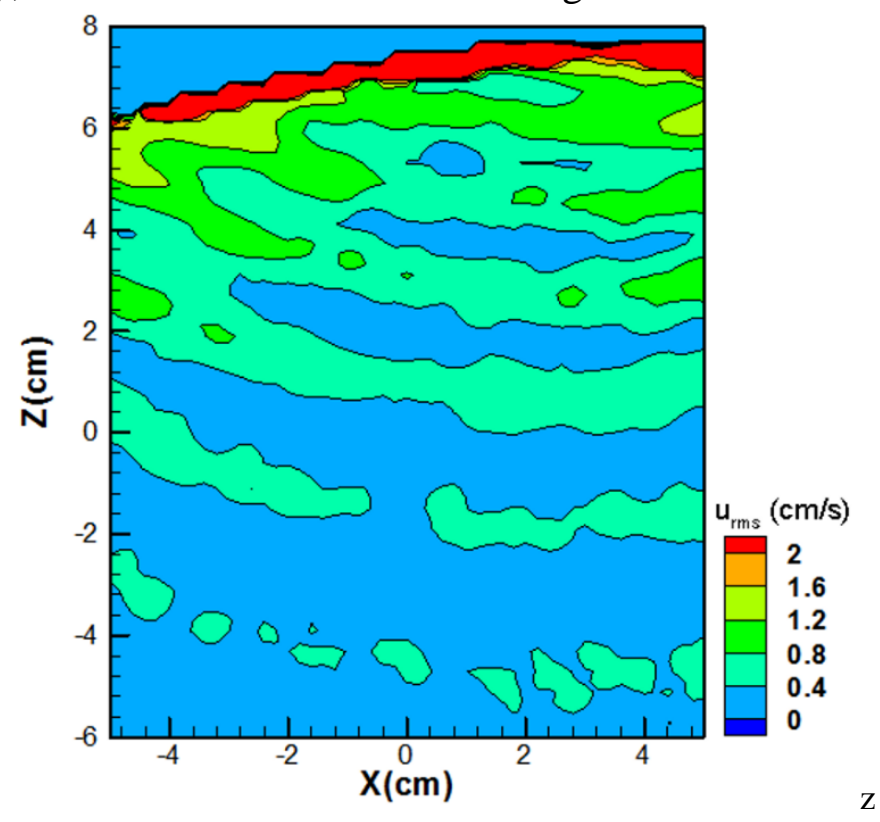

Fig. 7 RMS of horizontal water particle velocity field under the wave crest for case PR3

The root mean square (RMS) values for the case PR3 were presented in Fig. 7, which showed the relatively large value near the free surface due to the limitation of PIV technique near the boundary. The error rate of horizontal velocities of selected regular waves was estimated with the ratio of $u_{r m s}$ and the maximum velocity which was within $2 \%$ except very near the free surface region. 
The total acceleration are made of local and convective accelerations in Equations (5) and (6).

$$
\begin{aligned}
& \frac{d u}{d t}=\underbrace{\frac{\partial u}{\partial t}}_{\text {local }}+\underbrace{u \frac{\partial u}{\partial x}+w \frac{\partial u}{\partial z}}_{\text {convective }} \\
& \frac{d w}{d t}=\underbrace{\frac{\partial w}{\partial t}}_{\text {local }}+\underbrace{u \frac{\partial w}{\partial x}+w \frac{\partial w}{\partial z}}_{\text {convective }}
\end{aligned}
$$

where $d u / d t$ and $d w / d t$ represent total acceleration in horizontal and vertical directions, respectively. The local and convective acceleration fields were computed by applying the time and spatial central difference scheme to successive velocity profiles $(\Delta t=75 \mathrm{~ms})$ and each velocity profile in $\Delta x$ and $\Delta z(2.01 \mathrm{~mm})$, respectively. The acceleration was normalized by the gravity acceleration $g$. The horizontal local and convective accelerations of experimental results had a good agreement with those of the third-order Stokes wave theory through the depth for three regular waves in Fig. 8. But, for the case PR3, the horizontal local and convective accelerations became larger than the theory results getting closer to the wave crest. Note that the nonlinear effect of accelerations became more significant closer to the wave crest as the wave steepness increased.

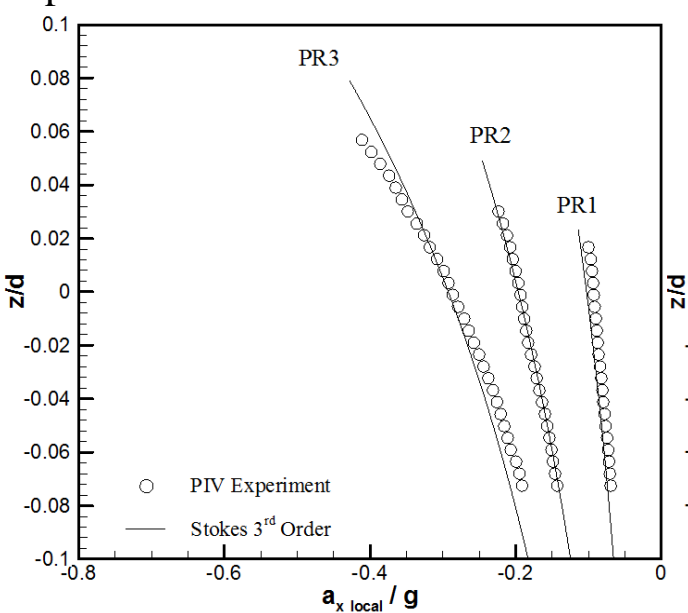

(a) Horizontal local accelerations

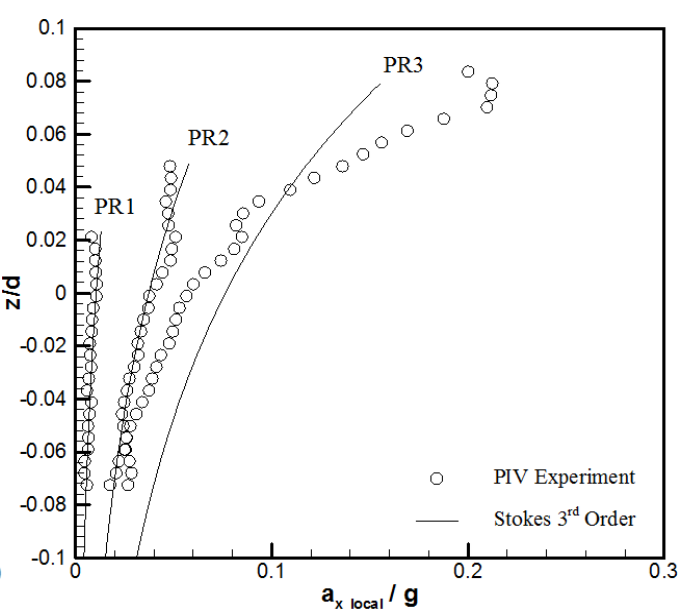

(b) Horizontal convective accelerations

Fig. 8 Horizontal local and convective acceleration under the wave crest (case PR3)

\section{Prediction methods of irregular wave kinematics}

Three prediction methods of the linear extrapolation, Wheeler stretching [33], and the modified stretching [34] were introduced to estimate the wave kinematics of irregular waves.

Using Fast Fourier Transform, a two-dimensional wave elevation can be decomposed into a series of component waves,

$$
\eta(x, t)=\sum_{i=1}^{N} A_{i} \cos \left(k_{i} t-\omega_{i} t+\phi_{i}\right)
$$


where $\mathrm{A}_{\mathrm{i}}, k_{i}, \omega_{i}, \phi_{i}$ and $N$ are the wave amplitude, wave number, wave circular frequency, wave phase, and the number of waves, respectively. The wave number and wave circular frequency are related to each other based on the linear dispersion relationship,

$$
\omega_{i}^{2}=g \cdot k_{i} \cdot \tan h\left(k_{i} h\right)
$$

where $h$ is the water depth. following,

According to the linear wave theory, the velocity components can be computed as

$$
\begin{aligned}
& u(x, z, t)=\sum_{i=1}^{N} A_{i} \frac{g \cdot k_{i}}{\omega_{i}} \cdot \frac{\cosh \left[k_{i} \cdot(h+z)\right]}{\cosh k_{i} h} \cdot \cos \left(k_{i} x-\omega_{i} t+\phi_{i}\right) \\
& w(x, z, t)=\sum_{i=1}^{N} A_{i} \frac{g \cdot k_{i}}{\omega_{i}} \cdot \frac{\sinh \left[k_{i} \cdot(h+z)\right]}{\cosh k_{i} h} \cdot \sin \left(k_{i} x-\omega_{i} t+\phi_{i}\right)
\end{aligned}
$$

The linear extrapolation method to predict the irregular wave kinematics up to the wave crest was that Equations (9) and (10) were modified above MWL with their linear Taylor expansion about MWL:

$$
u(x, z, t)=u(x, 0, t)+z \frac{\partial u}{\partial z}(x, 0, t), \quad \text { for } 0 \leq z \leq \eta
$$

The linear extrapolation method made the relatively large variation of the wave kinematics prediction over the mean water level (MWL) depending on the cut-off frequency of wave spectrum. Wheeler stretching method [33] was suggested to reduce the dependency on the cut-off frequency of the linear extrapolation method, which was made to map the vertical coordinate $z$ (from the seabed to the instantaneous free surface) onto the effective vertical coordinate $z_{e}$

$$
z_{e}=\frac{h \cdot\left(z_{a}-\eta\right)}{h+\eta}
$$

where $z_{a}$ is the actual vertical coordinate $\left(-h \leq z_{a} \leq H_{c}\right)$.

The horizontal and vertical water particle velocity, Equations (13) and (14) were developed as in the following,

$$
\begin{aligned}
& u(x, z, t)=\sum_{i=1}^{N} A_{i} \frac{g \cdot k_{i}}{\omega_{i}} \cdot \frac{\cosh \left[k_{i} \cdot\left(\frac{z_{a}+h}{1+\eta / h}\right)\right]}{\cosh k_{i} h} \cdot \cos \left(k_{i} x-\omega_{i} t+\phi_{i}\right) \\
& w(x, z, t)=\sum_{i=1}^{N} A_{i} \frac{g \cdot k_{i}}{\omega_{i}} \cdot \frac{\sinh \left[k_{i} \cdot\left(\frac{z_{a}+h}{1+\eta / h}\right)\right]}{\cosh k_{i} h} \cdot \cos \left(k_{i} x-\omega_{i} t+\phi_{i}\right)
\end{aligned}
$$

The linear extrapolation and Wheeler stretching methods were based on the linear wave theory and could be used for the wave kinematics prediction of a linear wave and a weakly nonlinear wave. Fig. 9 provides a brief concept for determining how the horizontal water 
velocity under the wave surface with the linear wave theory, the linear extrapolation, and Wheeler stretching. Velocity prediction of the linear extrapolation equaled to the linear wave theory value up to MWL and was increased with the vertical partial derivative in Equation (11) above MWL. The water particle velocity at the instantaneous free surface predicted by Wheeler stretching equaled to the linear wave theory value at MWL.

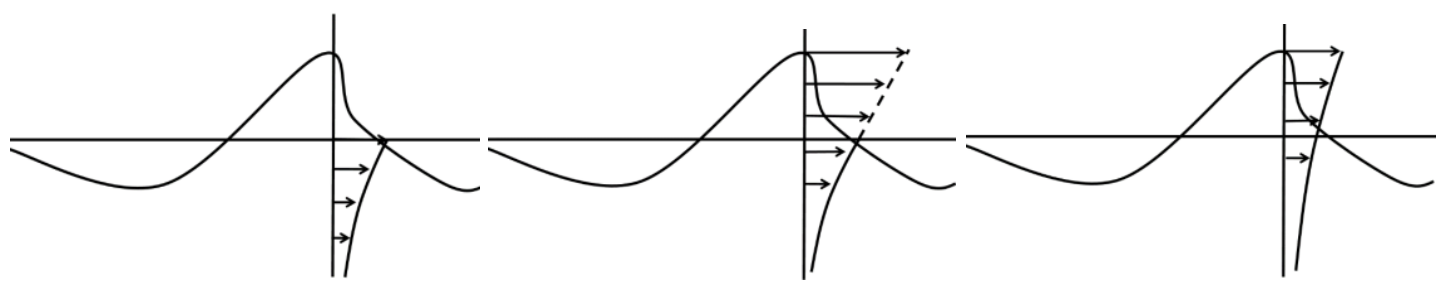

(a) Linear theory

(b)Linear extrapolation

(c) Wheeler Stretching

Fig. 9 Conceptual sketch of horizontal water particle velocity of approximate methods

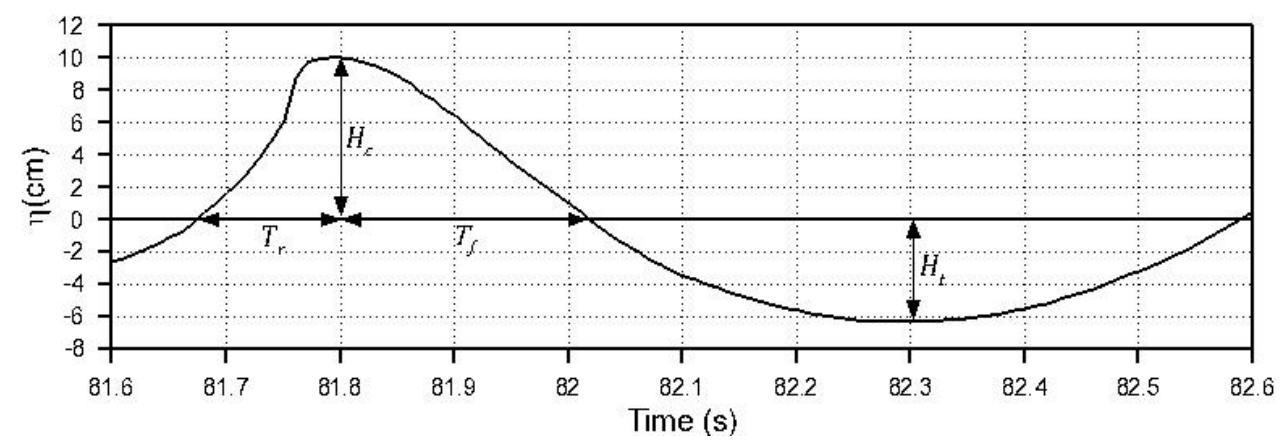

Fig. 10 Asymmetric factors of a rogue wave for the modified stretching method

Kim et al. [34] proposed the modified stretching model to take into account the asymmetries of the wave in prediction of the highly nonlinear wave kinematics. The asymmetric factors of the rogue wave are defined as shown in Fig. 10. The modified stretching method was given by

$$
z_{e}=a z_{a}^{3}+b z_{a}^{2}+c z_{a}+d \text { for }-h \leq z_{a} \leq H_{c}
$$

with

$$
\begin{aligned}
a & =\left[\left(-h+H_{c}\right)+k\left(h+H_{c}\right)\right] /\left(h+H_{c}\right)^{3} \\
b & =\left[-2\left(h^{2}-h H_{c}+H_{c}^{2}\right)-k\left(h+H_{c}\right)\left(H_{c}-2 h\right)\right] /\left(h+H_{c}\right)^{3} \\
c & =\left[H_{c}\left(H_{c}^{2}-H_{c} h+4 h^{2}\right)+k h\left(h+H_{c}\right)\left(h-2 H_{c}\right)\right] /\left(h+H_{c}\right)^{3} \\
d & =-\left\{h^{2} H_{c}\left[k h\left(h+H_{c}\right)+2 H_{c}\right]\right\} /\left(h+H_{c}\right)^{3} \\
k & =(2.00-\lambda) H_{t} / H \\
\lambda & =T_{f} / T_{r}
\end{aligned}
$$

When $H_{c} / H_{t} \leq 1.0, \lambda=1.0$, and when $H_{c} / H_{t}>1.0, \lambda=1.95$, where $z_{e}$ and $z_{a}$ were the effective vertical coordinate $\left(-h \leq z_{e} \leq 0\right)$ and the actual vertical coordinate $\left(-h \leq z_{z} \leq H_{c}\right)$, respectively. 
The velocity components of the highly nonlinear wave can be obtained through substituting the effective coordinate of Equation (15) into Equation (9) and (10) for a highly nonlinear wave following as:

$$
\begin{aligned}
& u(x, z, t)=\sum_{i=1}^{N} A_{i} \frac{g \cdot k_{i}}{\omega_{i}} \cdot \frac{\cosh \left[k_{i} \cdot\left(h+z_{e}\right)\right]}{\cosh k_{i} h} \cdot \cos \left(k_{i} x-\omega_{i} t+\phi_{i}\right) \\
& w(x, z, t)=\sum_{i=1}^{N} A_{i} \frac{g \cdot k_{i}}{\omega_{i}} \cdot \frac{\sinh \left[k_{i} \cdot\left(h+z_{e}\right)\right]}{\cosh k_{i} h} \cdot \sin \left(k_{i} x-\omega_{i} t+\phi_{i}\right)
\end{aligned}
$$

\section{Experimental results for rogue wave kinematics}

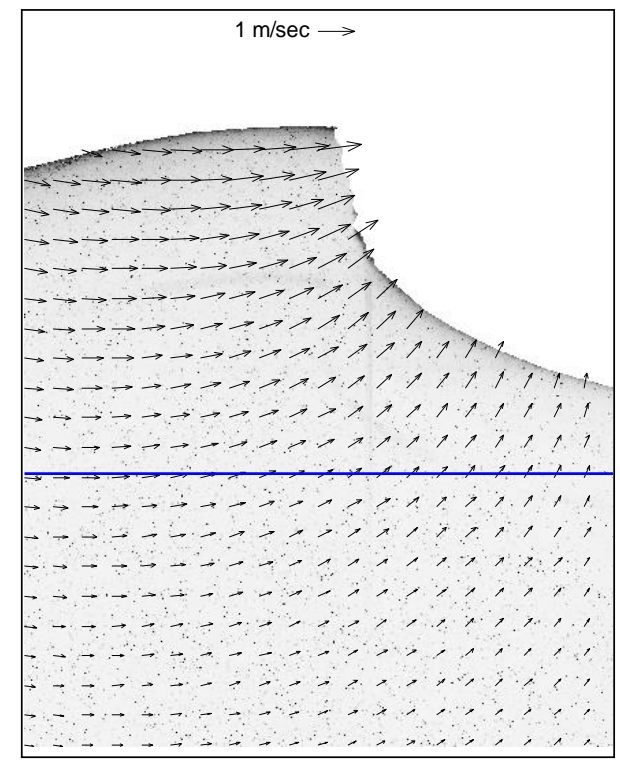

(a) Case PH3

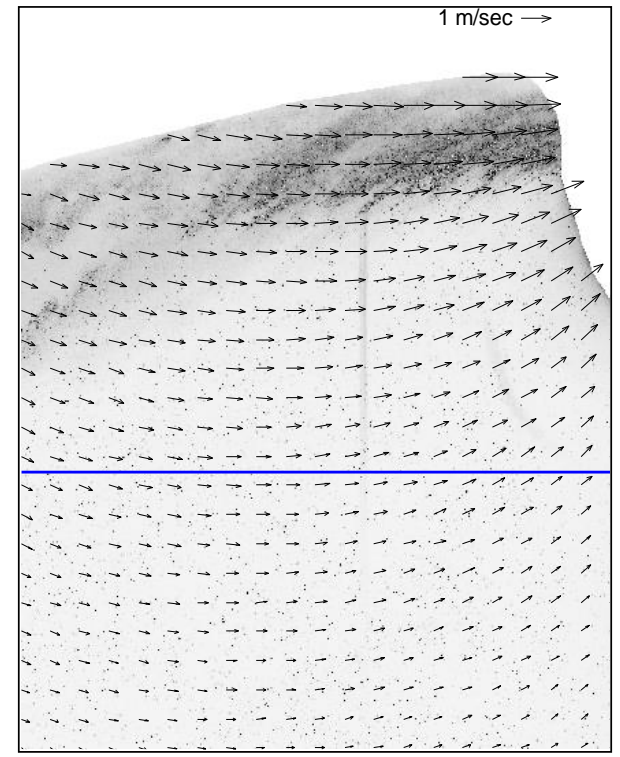

(b) Case PH4

Fig. 11 Velocity vector fields under the highest wave crest in cases PH3 and PH4

Four irregular wave trains were generated from the JONSWAP spectrum with the peak enhancement factor $\gamma(6.5)$ in 2-D wave tank (Table 2). The distortion method was applied to the irregular wave time series, and the highest wave crests of irregular wave trains in cases $\mathrm{PH} 3$ and PH4 met with two rogue wave criteria $\left(H / H_{s}>2.0\right.$ and $\left.H_{c} / H_{s}>1.25\right)$ in Table 2. Twenty eight instantaneous velocity fields were obtained with the time step of $75 \mathrm{~ms}$ including highest wave crests. The velocity profiles under the highest wave crest of cases PH3 and PH4 are shown on PIV snapshot images in Fig. 11. The solid line in the images indicated MWL and the velocity vectors under the free surface were well measured.

In Fig. 12 17, the measured and predicted velocity profiles were compared in normalized values; i.e., the vertical position $z$ normalized by water depth $d$ and velocities normalized by the phase velocity $V_{p}$ of the local wave period $(0.9 \mathrm{~s})$ at the highest wave crest shown in Fig. 4. The measured horizontal velocities were exponentially increased up to approximately $95 \%$ of the wave phase velocity. The measured vertical velocities were gradually increased up to $30 \%$ of the wave phase velocity over MWL and reduced to zero velocity at the wave crest because the water surface was the highest level at this moment. 


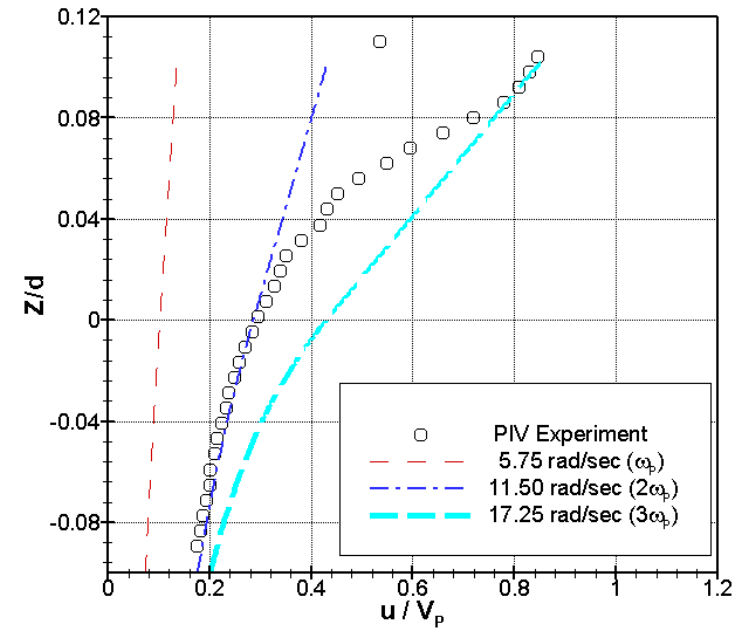

(a) Case PH3

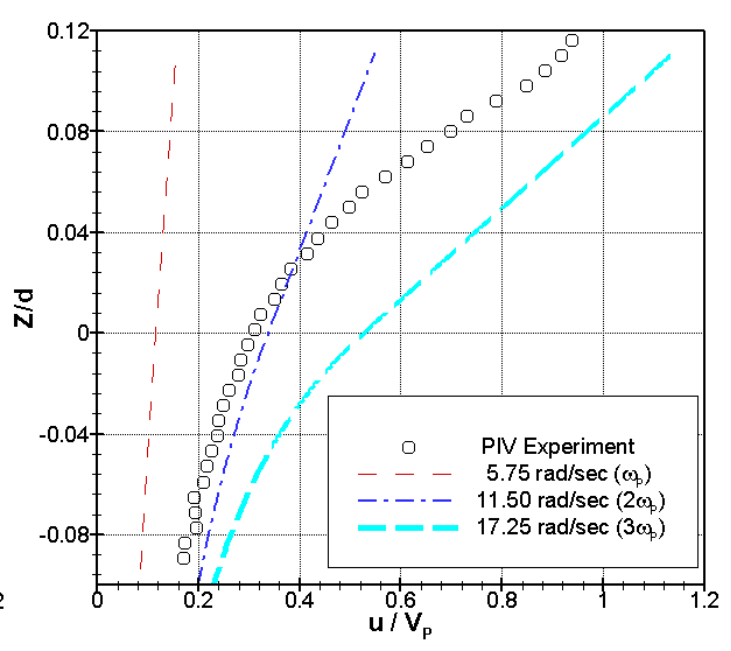

(b) Case PH4

Fig. 12 Comparison of measured horizontal velocity profiles and predictions of linear extrapolation varying cutoff frequency

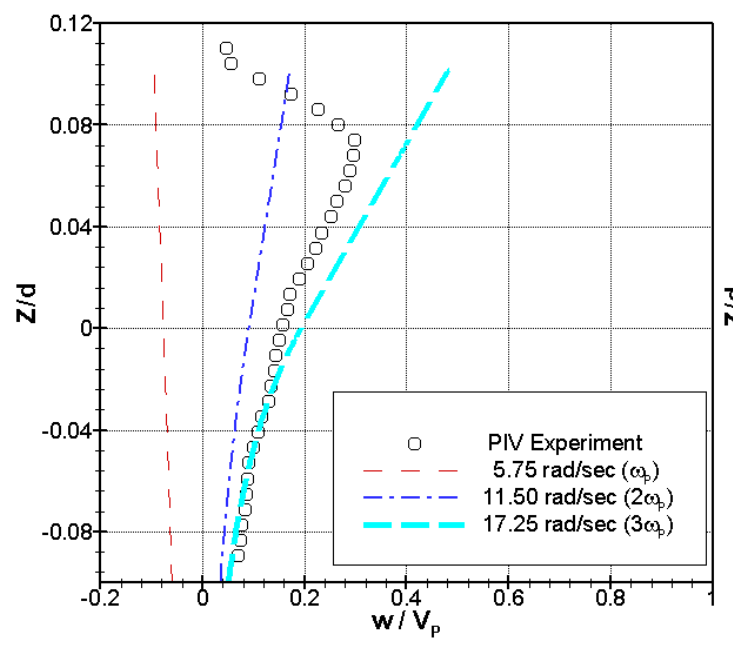

(a) Case PH3

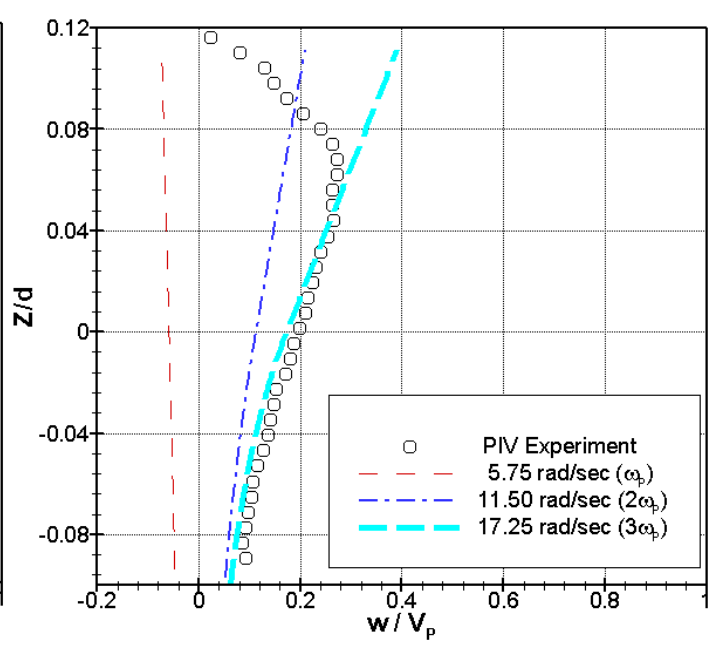

(b) Case PH4

Fig. 13 Comparison of measured vertical velocity profiles and predictions of linear extrapolation varying cut-off frequency

The wave kinematics estimated by three prediction methods of the linear extrapolation, Wheeler stretching, and the modified stretching with varying spectral cut-off frequency in order to investigate the cut-off frequency dependency. The linear extrapolation method overestimated horizontal water velocities at MWL with three times of peak wave period $\left(\omega_{p}\right)$ for cut-off frequency, but predicted similar magnitude and pattern for vertical velocities up to MWL. Because the linear extrapolation method assumed that the vertical partial derivative of a kinematic variable was constant above MWL as Equation (11), the predicted velocities were linearly increased up to the wave crest above MWL and were overestimated at three times of spectral peak frequency in comparison with the measured velocities over MWL as shown in Fig. 12 and 13. 
Hae Jin Choi, Seung Jae Lee, Hyo Jae Jo Gang Nam Lee, Kwang Hyo Jung

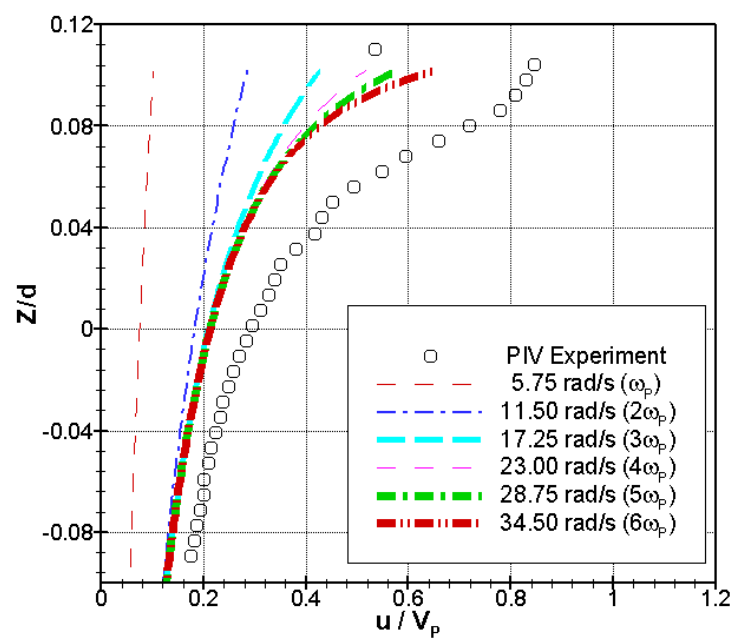

(a) Case PH3
Comparison Study of Experiments and Predictions of Wave Kinematics for Rogue Wave

Fig. 14 Comparison of measured horizontal velocity profiles and predictions of Wheeler stretching method varying cut-off frequency

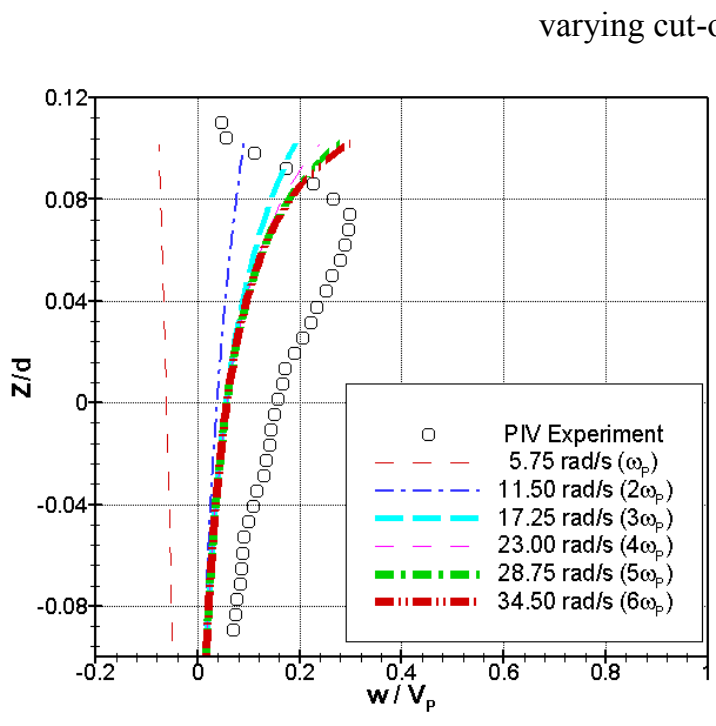

(a) Case PH3

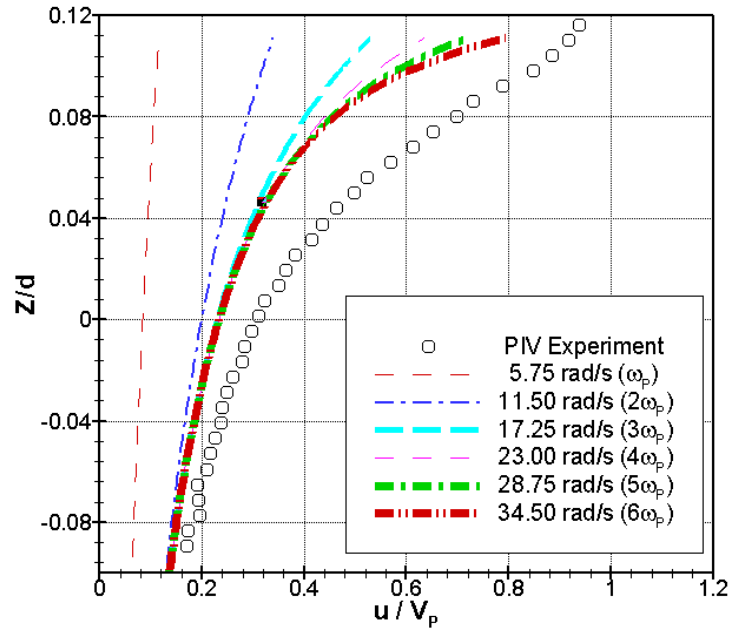

(b) Case PH4

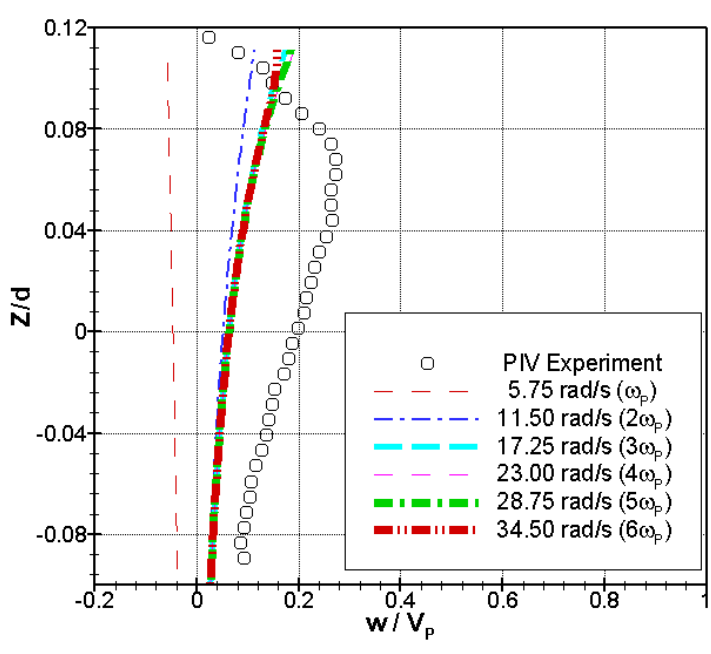

(b) Case PH4

Fig. 15 Comparison of measured vertical velocity profiles and predictions of Wheeler stretching method varying cut-off frequency

Wheeler stretching underestimated water velocities at MWL, because it was mapped the vertical coordinate $z$ onto the effective vertical coordinate and the same magnitude of water velocity predicted by the linear wave theory at MWL was stretched to the instantaneous free surface of the wave crest. It was clear that Wheeler stretching method reduced the cut-off frequency dependency for the sake of the underestimation of the water velocity magnitude above MWL in Fig. 14 and 15. 
Comparison Study of Experiments and Predictions of Wave Kinematics for Rogue Wave

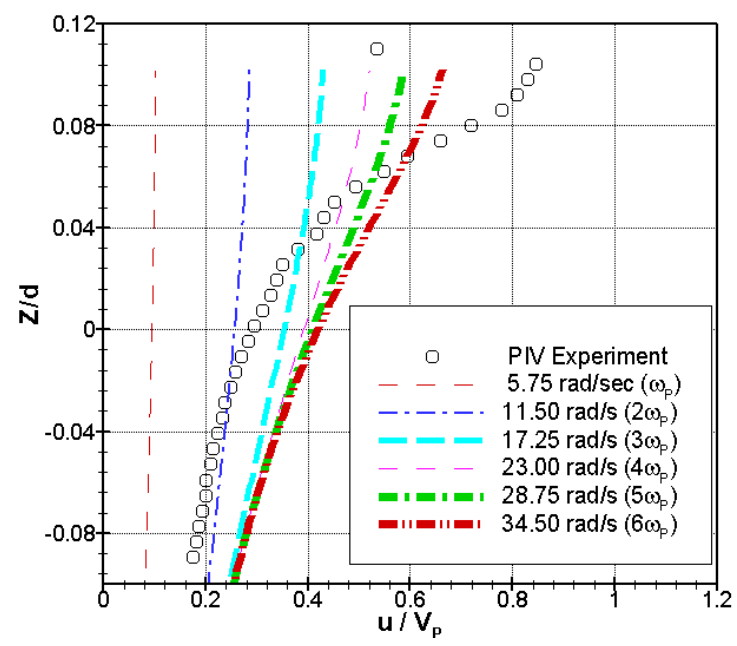

(a) Case PH3
Hae Jin Choi, Seung Jae Lee, Hyo Jae Jo Gang Nam Lee, Kwang Hyo Jung

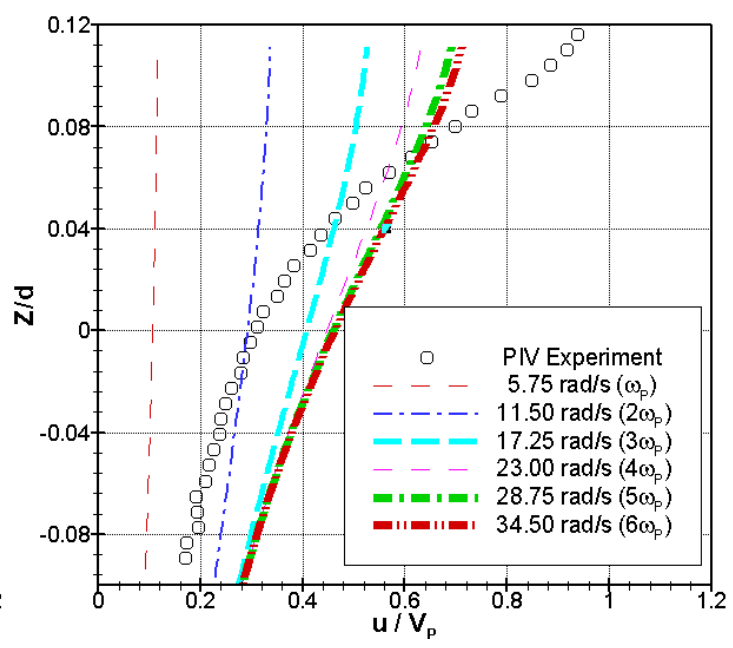

(b) Case $\mathrm{PH} 4$

Fig. 16 Comparison of measured horizontal velocity profiles and predictions of modified stretching method varying cut-off frequency

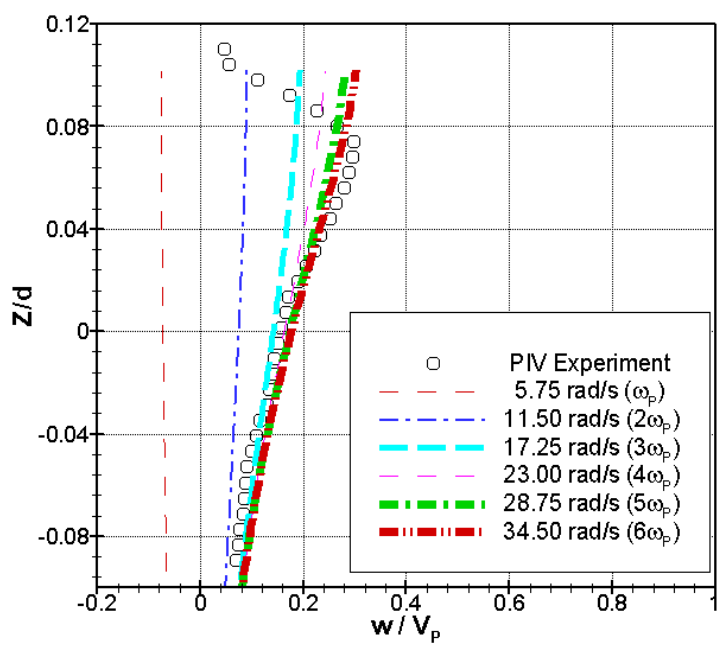

(a) Case PH3

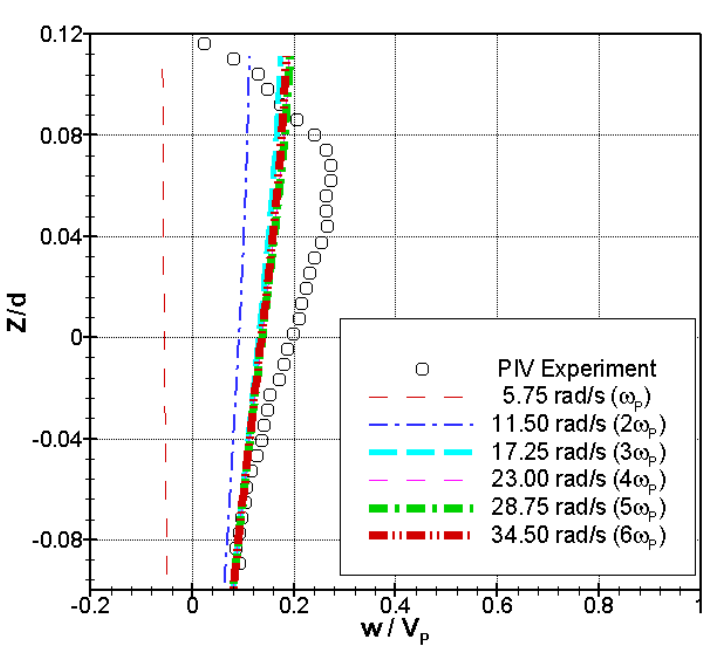

(b) Case PH4

Fig. 17 Comparison of measured vertical velocity profiles and predictions of modified stretching method varying

cut-off frequency

The modified stretching was proposed to predict the wave kinematics for highly nonlinear waves by Kim et al. [34], which calculated the horizontal and vertical velocity profiles under wave crests for rogue waves with varying the cut-off frequency and compared with experimental results in Fig. 16 and 17, respectively. It showed less sensitive to be chosen the cut-off frequency than results of the linear extrapolation method, and the better agreement with experimental results over MWL than the prediction of Wheeler stretching. From the investigation of the sensitivity of cut-off frequency in comparison with experimental results, the linear extrapolation method was able to predict the water velocity profile under the highest wave crest in irregular wave train with three time of spectral peak wave frequency for cut-off frequency, and predictions of Wheeler and modified stretching methods had a good agreement with the experimental results with higher cut-off frequency than four or five times of spectral peak wave frequency. 
Hae Jin Choi, Seung Jae Lee, Hyo Jae Jo Gang Nam Lee, Kwang Hyo Jung
Comparison Study of Experiments and Predictions of Wave Kinematics for Rogue Wave

Local accelerations under the wave crest of rogue waves were shown for cases $\mathrm{PH} 3$ and PH4 in Fig. $18 \sim 23$. The local acceleration was calculated from consecutive velocity profiles ( $\Delta t=75 \mathrm{~ms}$ ) taken by PIV system under the highest wave crest of irregular wave trains with the central difference method. Because the central difference method was able to be applied below the velocity vector at the lower free surface among the previous and following PIV images, local accelerations were calculated only below the water level for lower wave crest of them. But, note that the results of local acceleration profiles calculated with the measured velocities were reasonably agreed with the Stokes $3^{\text {rd }}$ order wave theory for steep regular waves in Fig. 8 . The horizontal local acceleration could be reached up to $0.8 \mathrm{~g} \sim 1.0 \mathrm{~g}$ and the vertical local acceleration up to $-0.8 g \sim-1.0 \mathrm{~g}$ at near wave crest for PH3 and PH4 if it was extended up to the wave crest level.

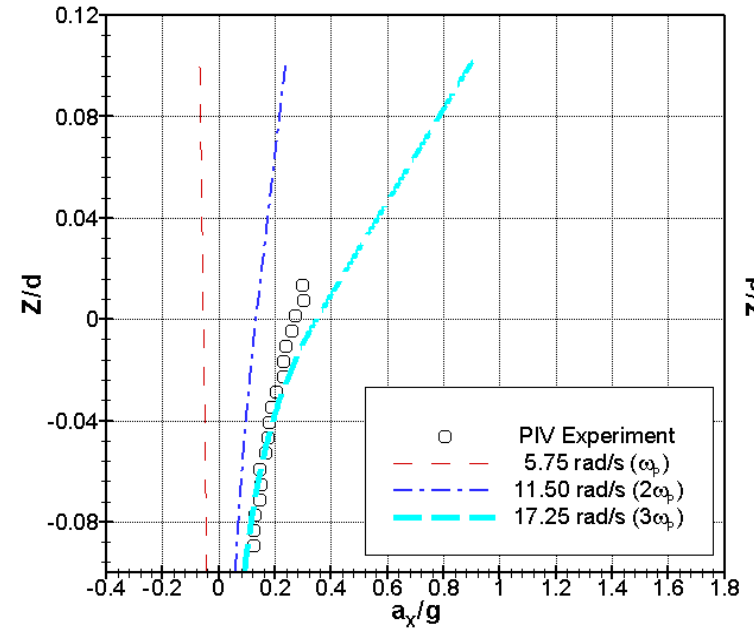

(a) Case PH3

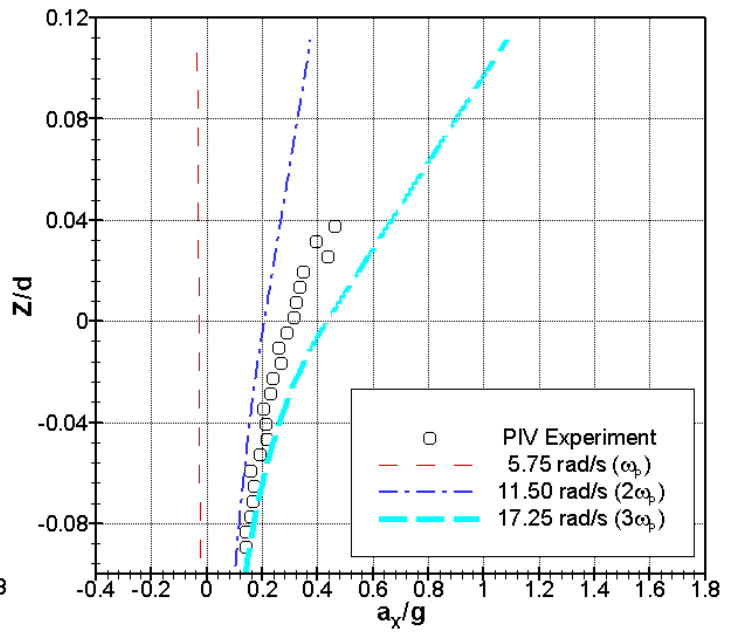

(b) Case PH4

Fig. 18 Comparison of measured horizontal local accelerations profiles and predictions of linear extrapolation varying cut-off frequency

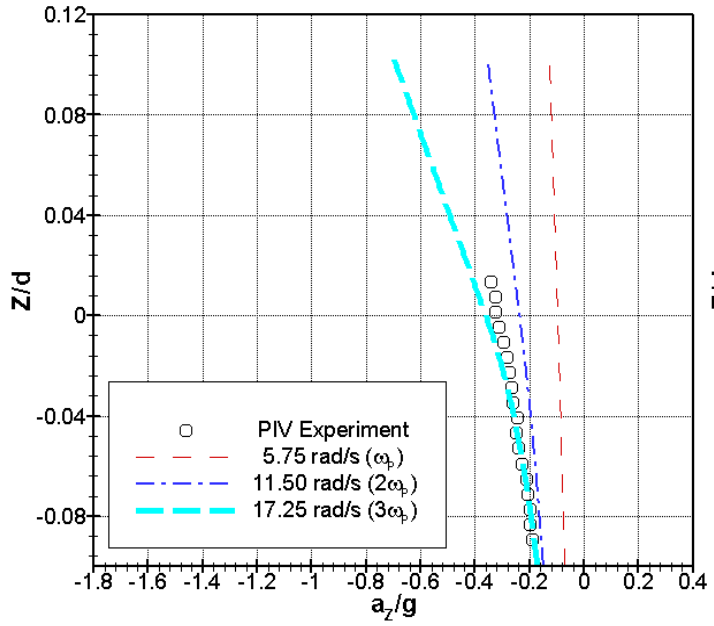

(a) Case PH3

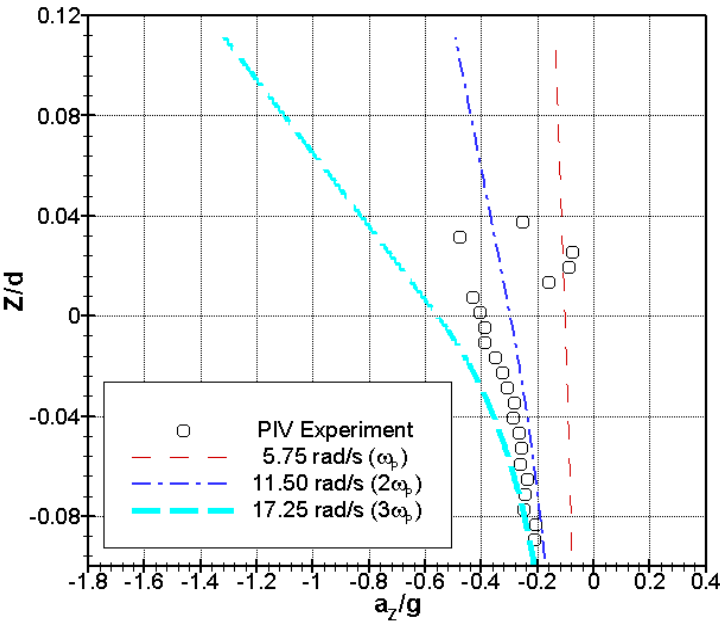

(b) Case PH4

Fig. 19 Comparison of measured vertical local accelerations profiles and predictions of linear extrapolation varying cut-off frequency 
Comparison Study of Experiments and Predictions of Wave Kinematics for Rogue Wave

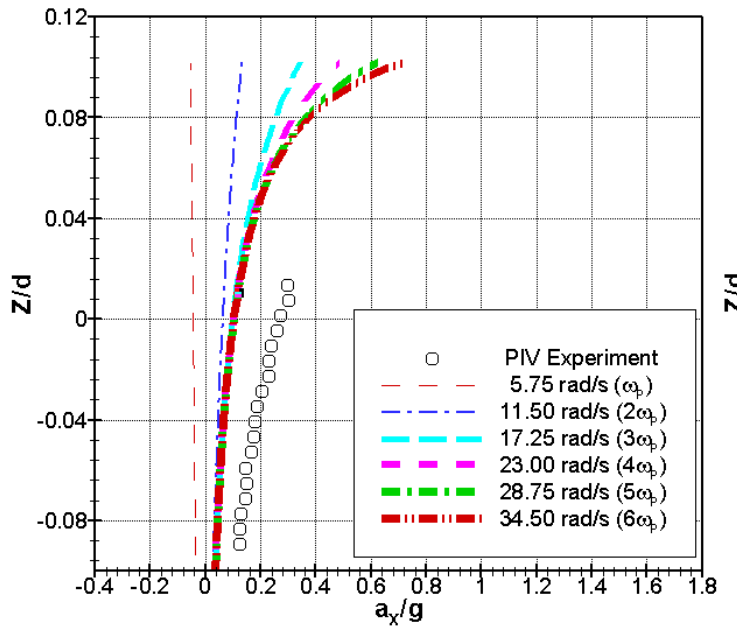

(a) Case PH3
Hae Jin Choi, Seung Jae Lee, Hyo Jae Jo Gang Nam Lee, Kwang Hyo Jung

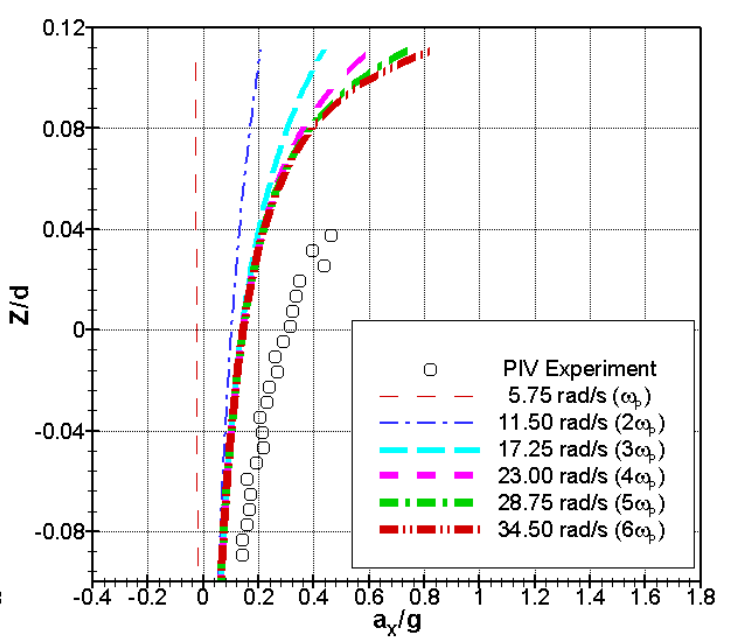

(b) Case $\mathrm{PH} 4$

Fig. 20 Comparison of measured horizontal local accelerations profiles and predictions of Wheeler stretching method varying cut-off frequency

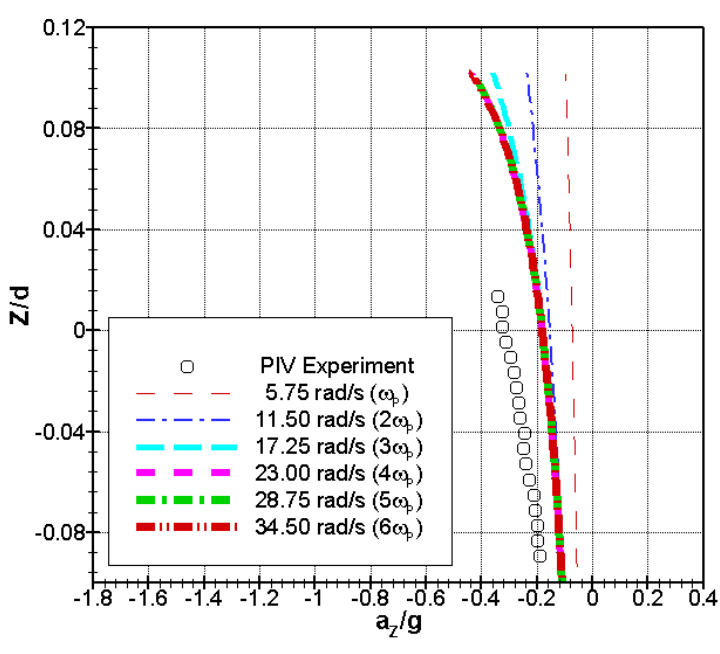

(a) Case PH3

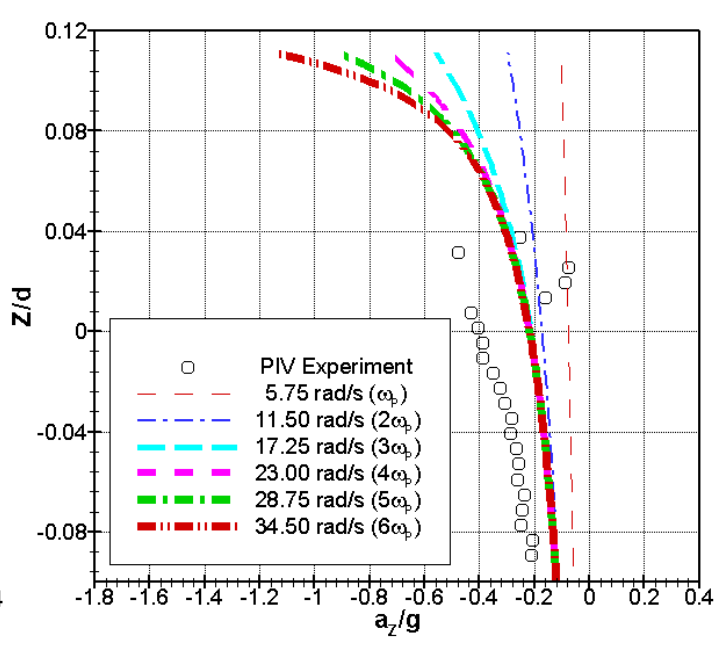

(b) Case PH4

Fig. 21 Comparison of measured vertical local accelerations profiles and predictions of Wheeler stretching

method varying cut-off frequency

Linear extrapolation method was over-predicted the local acceleration with three times of spectral peak frequency for cut-off frequency in comparison with those computed from measured velocities as shown in Fig. 18 and 19 because of the constant vertical partial derivative above MWL. Local accelerations predicted by Wheeler stretching method were exponentially increased up to the wave crest with the higher cut-off frequency and had a less sensitivity for increasing the cut-off frequency in Fig. 20 and 21. However, it underestimated the horizontal and vertical local accelerations across the overall water depth. 
Hae Jin Choi, Seung Jae Lee, Hyo Jae Jo Gang Nam Lee, Kwang Hyo Jung

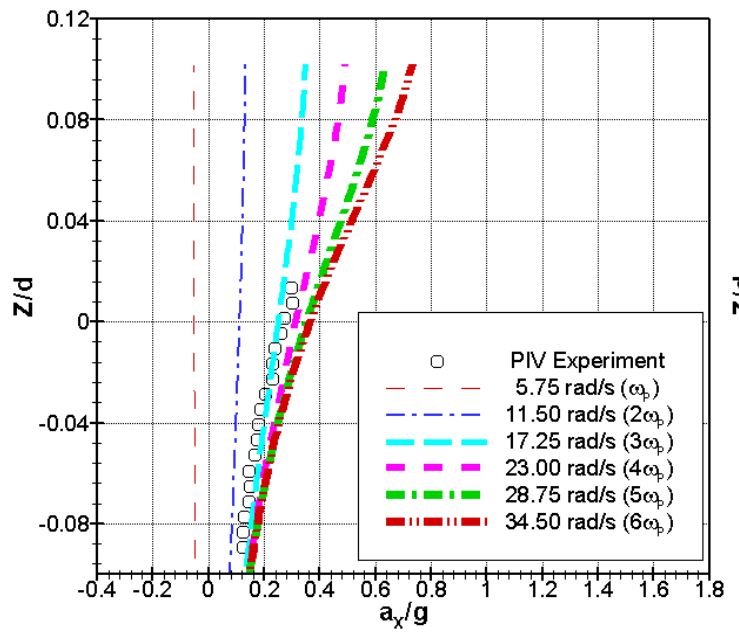

(a) Case PH3
Comparison Study of Experiments and Predictions of Wave Kinematics for Rogue Wave

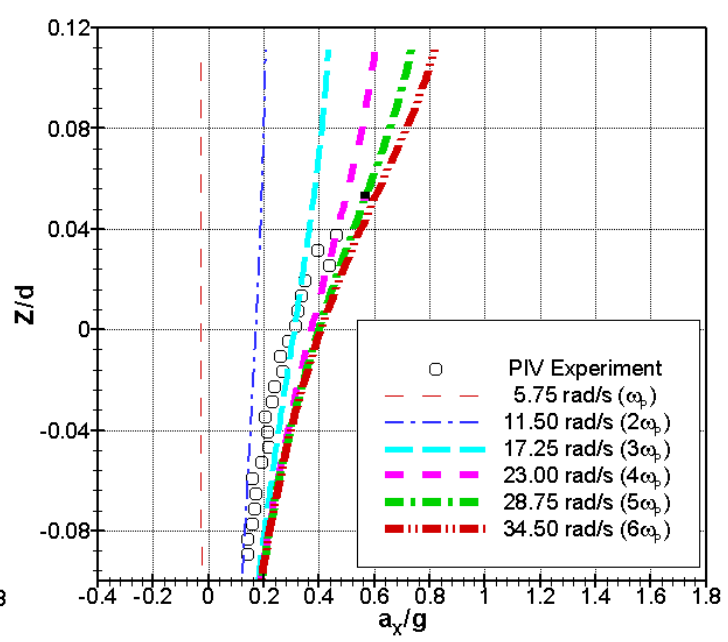

(b) Case PH4

Fig. 22 Comparison of measured horizontal local accelerations profiles and predictions of Modified stretching method varying cut-off frequency

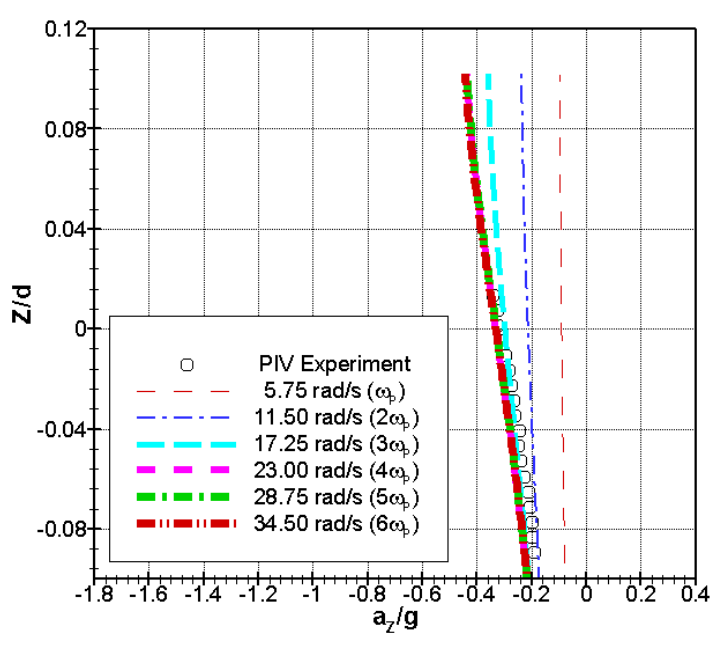

(a) Case PH3

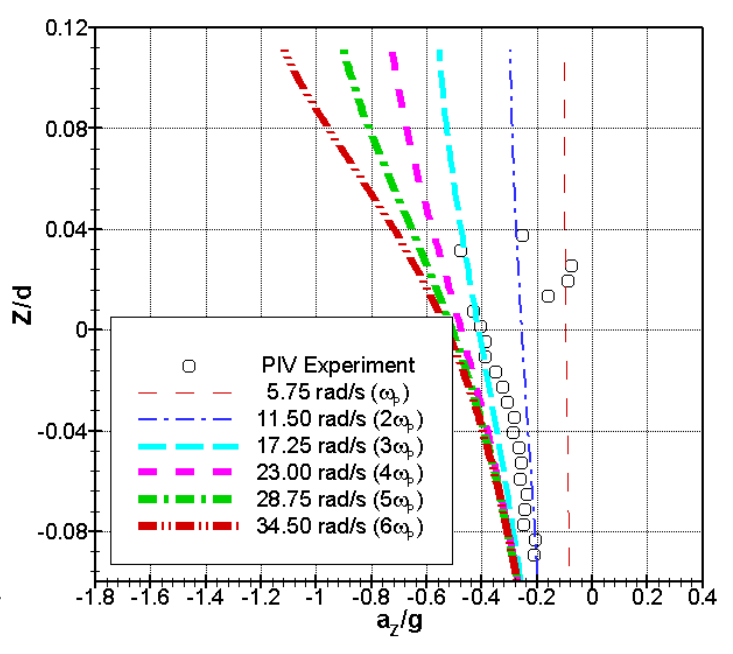

(b) Case PH4

Fig. 23 Comparison of measured vertical local accelerations profiles and predictions of Modified stretching

method varying cut-off frequency

In Fig. 22 and 23, local accelerations predicted by the modified stretching were compared with experimental results. With varying the cut-off frequency, they were reasonably increased across the water depth and higher increasing rate over MWL. The modified stretching method had a good agreement with horizontal and vertical local accelerations of experimental results at three to four times of spectral peak frequency for the cut-off frequency.

In comparison of measured velocity and local acceleration profiles and predictions of three methods in varying cut-off frequency, it showed similar trends that the modified stretching had less sensitive for the higher cut-off frequency than the linear extrapolation method, and quantitatively a better agreement with experimental results across the water depth rather than Wheeler stretching method. 
Comparison Study of Experiments and Predictions of Wave Kinematics for Rogue Wave

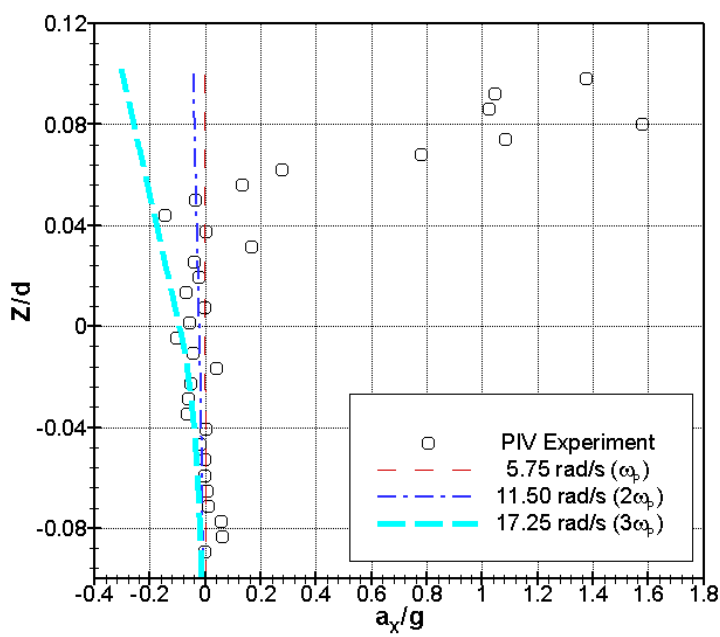

(a) Case PH3
Hae Jin Choi, Seung Jae Lee, Hyo Jae Jo Gang Nam Lee, Kwang Hyo Jung

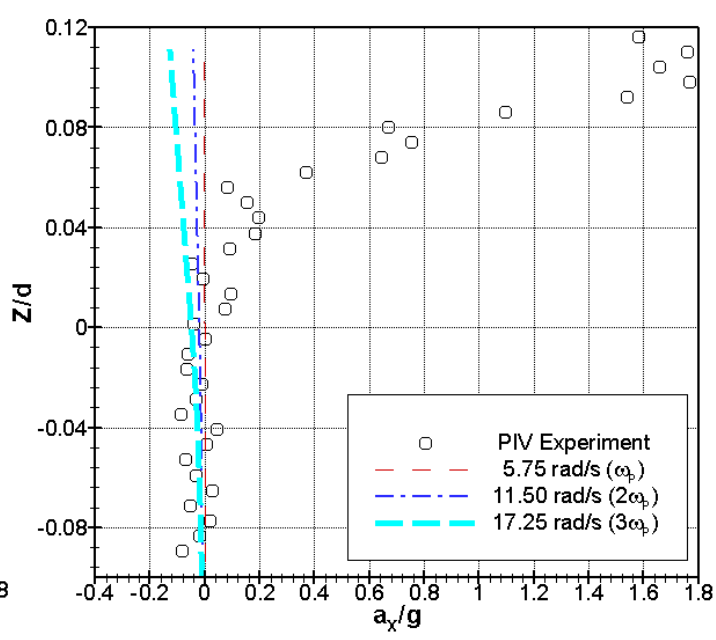

(b) Case PH4

Fig. 24 Comparison of measured horizontal convective accelerations profiles and predictions of linear extrapolation varying cut-off frequency

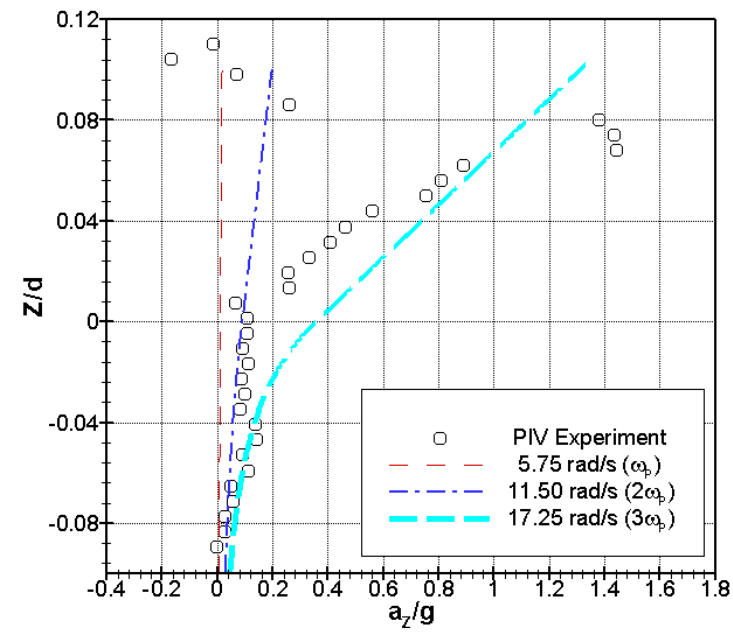

(a) Case PH3

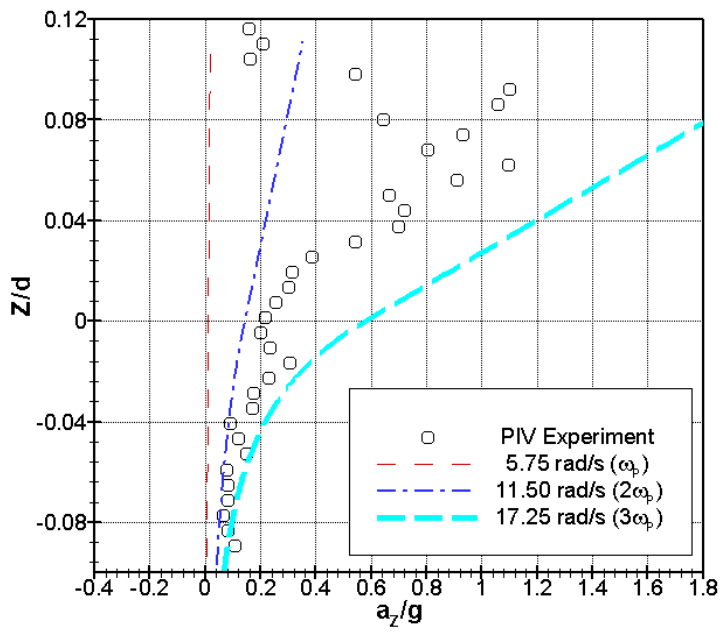

(b) Case PH4

Fig. 25 Comparison of measured vertical convective accelerations profiles and predictions of linear extrapolation varying cut-off frequency 
Hae Jin Choi, Seung Jae Lee, Hyo Jae Jo Gang Nam Lee, Kwang Hyo Jung

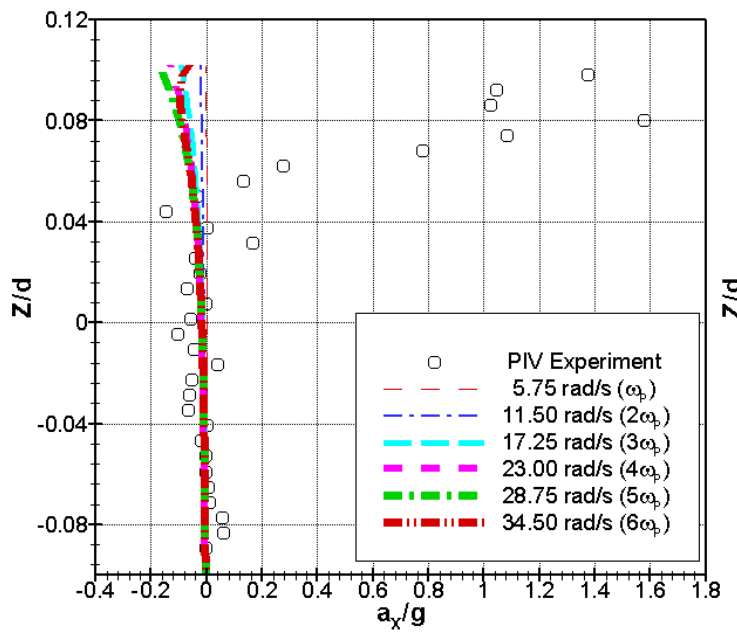

(a) Case PH3
Comparison Study of Experiments and Predictions of Wave Kinematics for Rogue Wave

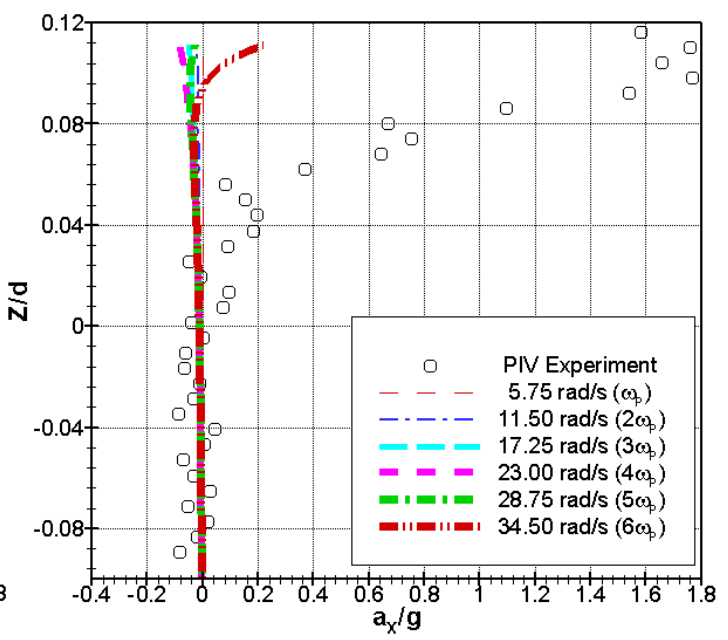

(b) Case $\mathrm{PH} 4$

Fig. 26 Comparison of measured horizontal convective accelerations profiles and predictions of Wheeler stretching method varying cut-off frequency

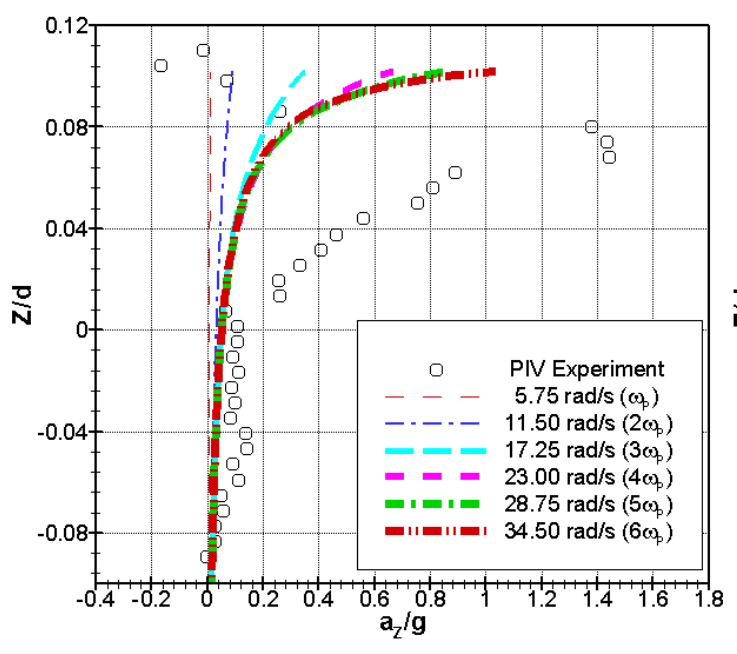

(a) Case PH3

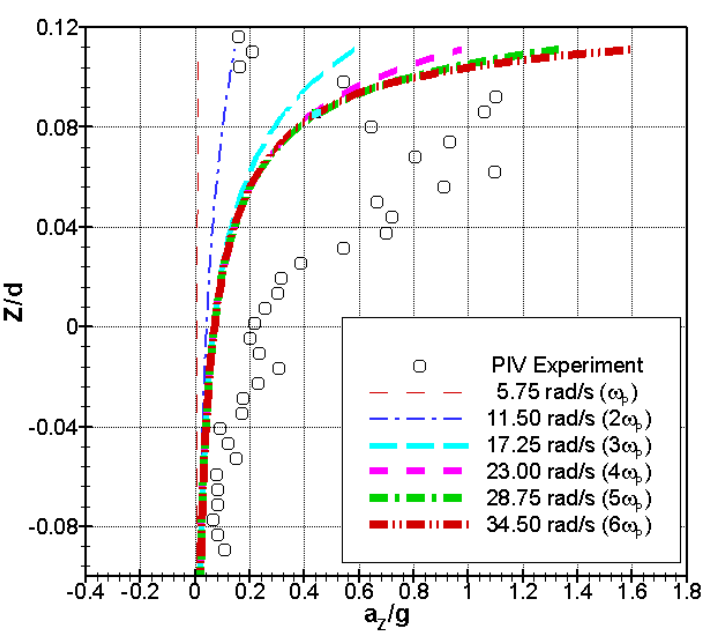

(b) Case PH4

Fig. 27 Comparison of measured vertical convective accelerations profiles and predictions of Wheeler stretching method varying cut-off frequency 
Comparison Study of Experiments and Predictions of Wave Kinematics for Rogue Wave

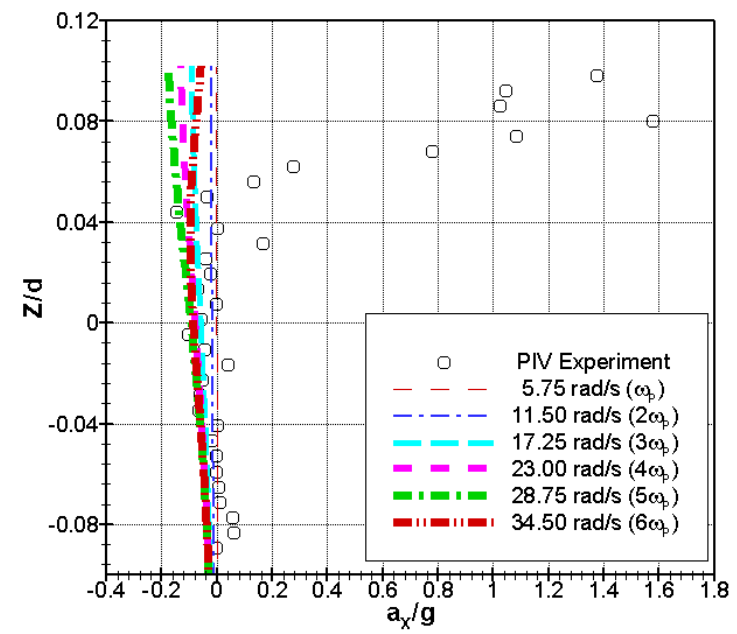

(a) Case PH3
Hae Jin Choi, Seung Jae Lee, Hyo Jae Jo Gang Nam Lee, Kwang Hyo Jung

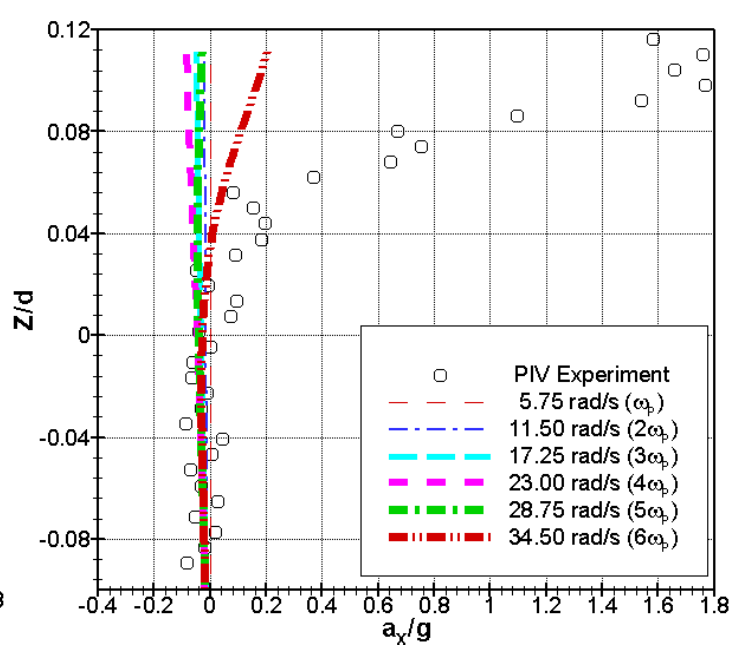

(b) Case PH4

Fig. 28 Comparison of measured horizontal convective accelerations profiles and predictions of Modified stretching method varying cut-off frequency

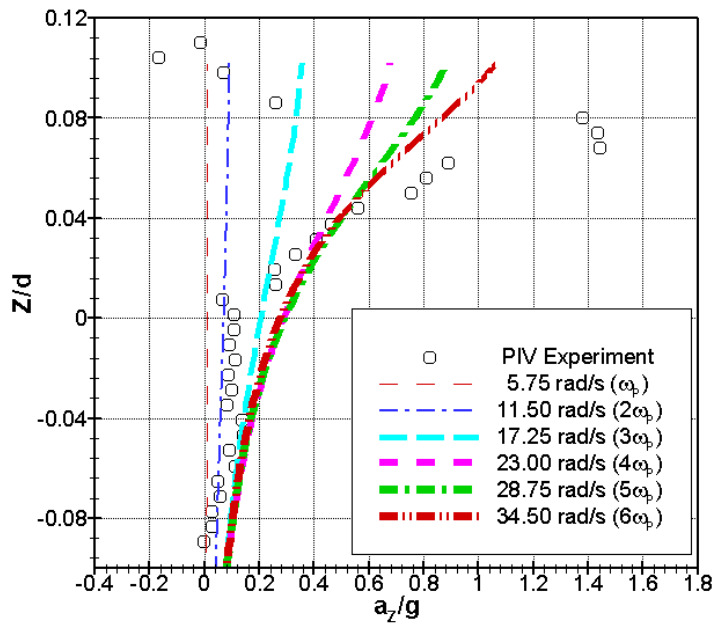

(a) Case PH3

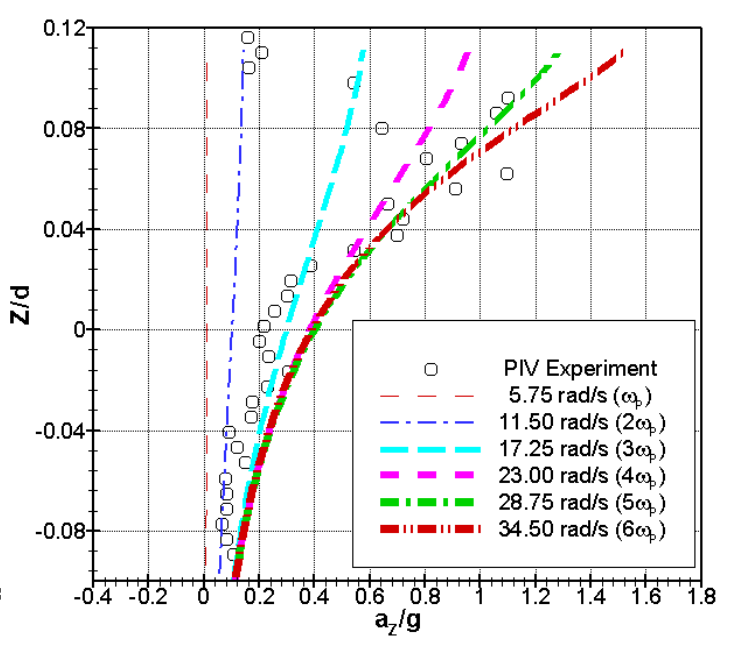

(b) Case PH4

Fig. 29 Comparison of measured vertical convective accelerations profiles and predictions of Modified

stretching method varying cut-off frequency

In Fig. 24 29, horizontal and vertical convective accelerations for cases PH3 and PH4 calculated with the application of the central difference scheme in the spatial resolution, 2.72 $\mathrm{mm}$, were compared with predicted results of three methods. The convective accelerations were very small and nearly close to zero under MWL and were rapidly increased over the gravity acceleration in both directions. Even though the experimental results were some scattered, those increasing patterns were consistent for all irregular wave conditions. The horizontal accelerations predicted by three methods were well agreed with experimental results below MWL, but showed the opposite sign with experimental results over MWL, although those magnitudes were small. However, the vertical convective accelerations were predicted in the pattern of linearly increasing over MWL by the linear extrapolation method and suddenly increasing near the wave crest with higher cut-off frequency by Wheeler stretching. Modified stretching method estimated the vertical convective acceleration in the similar magnitude and 
increasing pattern up to the wave crest with higher cut-off frequency overall water depth. The large discrepancy in convective accelerations, especially in horizontal direction, between experimental results and prediction methods could be caused by the limitation of three method based on the linear wave theory.

\section{Conclusions}

A series of experiments were conducted in 2-D wave tank to simulate the rogue waves and measure water wave kinematics including velocity and acceleration profiles. PIV technique was applied to measure the velocities under the free surface of regular and irregular waves. To verify the image acquisition and analysis methods of PIV, three different wave heights of regular wave having $0.9 \mathrm{~s}$ wave period were tested and those velocities and accelerations were compared with results of Stokes $3^{\text {rd }}$ order theory, which showed a good agreement up to the wave crest. To generate the rogue wave in the 2-D wave tank, three steps of amplitude distortion, time distortion, and crest distortion techniques were applied for irregular wave train using JONSWAP spectrum with a peak enhancement factor of 6.5. Two of four irregular wave trains were satisfied with the two rouge wave criteria $\left(H / H_{s}>2.0\right.$ and $\left.H_{c} / H_{s}>1.25\right)$. Three different prediction methods of linear extrapolation, Wheeler stretching, and modified stretching were applied to estimate water wave kinematics under the highest water elevation of irregular wave trains and to investigate the sensitivity of cut-off frequency with six different spectral peak frequencies.

Horizontal water velocity measured under the highest wave crest was increased up to $95 \%$ of the wave phase velocity calculated with the local wave period near the free surface. And, the maximum velocity in the vertical direction was measured at the middle location of the wave crest and MWL with approximately $30 \%$ of wave phase velocity. Although the local acceleration was not calculated with measured velocities up to the wave crest of rogue wave, its magnitude could be estimated approximately to the gravitation acceleration in both directions. The horizontal and vertical convective accelerations calculated with measured velocities were very small under MWL and were rapidly increased over the gravity acceleration.

The velocity and acceleration profiles had a significant effect of the cut-off frequency over MWL. Linear extrapolation predicted the water wave kinematics with strong sensitivity of cut-off frequency and overestimated them to be larger than experimental results except the horizontal local acceleration due to the assumption of constant vertical partial derivative of a kinematic variable above MWL. The water wave kinematics predicted by Wheeler stretching converged well with measurements as the cut-off frequency became higher, but underestimated those magnitudes to be smaller than the measurement results across the water depth. The modified stretching was relatively less sensitive for choosing the higher cut-off frequency than the linear extrapolation and better agreement with experimental results above MWL than other prediction methods. From the investigation of the cut-off frequency dependence on the water wave kinematics of water velocity and acceleration under the wave crest for rogue waves, it can be suggested that the cut-off frequency for the prediction of the water wave kinematics should be three times of spectral peak wave frequency for the linear extrapolation and higher frequency than four times of spectral peak wave frequency for Wheeler stretching and the modified stretching.

\section{ACKOWLEDGEMENTS}

This work was supported by the National Research Foundation of Korea (NRF) grant funded by the Ministry of Science (Republic of Korea) through Global Core Research Center for Ships and Offshore Plants (No. 2011-0030013) and the Ministry of Trade, Industry \& Energy (Republic of Korea) under Industrial Technology Innovation Program (No. 10063405) 
Comparison Study of Experiments and Predictions of

Wave Kinematics for Rogue Wave
Hae Jin Choi, Seung Jae Lee, Hyo Jae Jo

Gang Nam Lee, Kwang Hyo Jung

\section{REFERENCES}

[1] Kjeldsen, P., 2001. A sudden disaster-in extreme waves. Rogue Waves 2000, Brest.

[2] Met Office. 1996. Hurricane 'Luis', the Queen Elizabeth 2, and a rogue wave. Marine Observer. 66(333), 134-137.

[3] Mastroianni, M., 2015. Giant wave hits semester at sea ship. Available from: http://pittnews.com/article/33203/archives/giant-wave-hits-semester-at-sea-ship/

[4] Lemire, J., 2005 Apr 17. Freak wave rocks cruise. Available from: http://www.nydailynews.com/archives/news/freak-wave-rocks-cruise-70-footer-hits-n-y-bound-shiparticle-1.622703 ()

[5] Clauss, G.F., 2002. Dramas of the sea: episodic waves and their impacts on offshore structures. Applied Ocean Research. 24, 147-161. https://doi.org/10.1016/S0141-1187(02)00026-3.

[6] Rosenthal, W., Lehner, S., 2004. Results from the MAXWAVE project. Proc the 23th OMAE conference, Vancouver, Canada.

[7] Draper, D., 1965. Freak ocean waves. Marine Observer. 35, 193-195.

[8] Dean, R.G., 1990. Freak wave: a possible explanation. In: Tørum A, Gudmestad, OT. (Eds.). Water Wave Kinematics; Kluwer, the Netherlands. https://doi.org/10.1007/978-94-009-0531-3 39.

[9] Haver, S., 2001. Evidences of the existence of freak waves. Rogue Waves 2000, Brest.

[10] Olagnon, M., van Iseghem S. 2001. Some cases of observed rogue waves and an attempt to characterize their occurrence conditions. Rogue Waves 2000, Brest.

[11] Kharif, C., Pelinovsky, E., Talipova, T., Slunyaev, A., 2001. Focusing of nonlinear wave groups in deep water. JETP Letters. 73(4), 170-175. https://doi.org/10.1134/1.1368708.

[12] Smith, S.F., Swan, S., 2002. Extreme two-dimensional water waves: an assessment of potential design solutions. Ocean Engineering, 29(4), 387-416. https://doi.org/10.1016/S0029-8018(01)00028-2.

[13] Wu, C.H., Nepf, H., 2002. Breaking criteria and energy losses for three-dimensional wave breaking. Geophysical Research. 107 (c10), (41)1-18.

[14] Peregrine, D.H., 1976. Interaction of water waves and currents. Advances in Applied Mechanics. 16, 9 117. https://doi.org/10.1016/S0065-2156(08)70087-5.

[15] Smith, R., 1976. Giant waves. J of Fluid Mechanics. 77, 417-431.

[16] Lavrenov, I.V., 1998. The wave energy concentration at the Agulhas current off South Africa. J of Natural Hazards. 17, 117-127.

[17] Tulin, M.P., Waseda, T., 1999. Laboratory observations of wave group evolution, including breaking effects. J of Fluid Mechanics. 378, 197-232. https://doi.org/10.1017/S002211207600219X.

[18] Trulsen, K., Dysthe, K.B., 1997. Freak waves-A three dimensional wave simulation. Proc of the 21th Symposium on Naval Hydro-dynamics: The National Academy of Sciences, Washington, D.C.

[19] Dysthe, K.B., Trulsen, K., 1999. Note on breather type solutions of the NLS as a model for freak-waves. Physica Scripta. T82, 48-52. https://doi.org/10.1238/Physica.Topical.082a00048.

[20] Henderson, K.L., Peregrine, D.H., Dold, J.W., 1999. Unsteady water wave modulations: Fully nonlinear solutions and comparison with the nonlinear Schrödinger equation. Wave Motion. 29, 341-361. https://doi.org/10.1016/S0165-2125(98)00045-6.

[21] Osborne, A.R., Onorato, M., Serio, M., 2000. The nonlinear dynamics of rogue waves and holes in deepwater gravity wave trains. J of Physics Letters A. 274, 386-393. https://doi.org/10.1016/S03759601(00)00575-2.

[22] Onorato, M., Osborne, A.R., Serio, M., 2002. Extreme wave events in directional, random oceanic sea state. J of Physics of Fluids. 14(4), L25-L28. https://doi.org/10.1063/1.1453466.

[23] Lu, W., Yang, J., Tao, L., 2016. Numerical study of the energy structure of super rogue waves. Ocean engineering. 113, 295-307. https://doi.org/10.1016/j.oceaneng.2015.11.006.

[24] Zhang. H.D., Soares, C.G., 2016. Ship responses to abnormal waves simulated by the nonlinear Schrödinger equation. Ocean engineering. 119, 143-153. https://doi.org/10.1016/j.oceaneng.2016.03.064.

[25] Mori, N., Yasuda, T., 2001. Effects of high-order nonlinear wave-wave interactions on gravity waves. Rogue Waves 2000, Brest.

[26] Janssen, P.A.E.M., 2003. Nonlinear four-wave interactions and freak waves. J of Physical Oceanography. 33, 863-884. https://doi.org/10.1175/1520-0485(2003)33<863:NFIAFW>2.0.CO;2. 
Hae Jin Choi, Seung Jae Lee, Hyo Jae Jo Gang Nam Lee, Kwang Hyo Jung
Comparison Study of Experiments and Predictions of Wave Kinematics for Rogue Wave

[27] Grue, J., 2002. On four highly nonlinear phenomena in wave theory and marine hydrodynamics. Applied Ocean Research. 24, 261-274. https://doi.org/10.1016/S0141-1187(03)00006-3.

[28] Clamond, D., Grue, J., 2002. Interaction between envelop solitons as a model for freak wave formulations-part 1: Long time interaction. Comptes Rendus Mecanique. 330, 575-580. https://doi.org/10.1016/S1631-0721(02)01496-1.

[29] Wu, C.H., Yao, A., 2004. Laboratory measurements of limiting freak waves on currents Geophysical Research. 109(c12002), 1-18. https://doi.org/10.1029/2004JC002612.

[30] Zou, J., Kim, C.H., 2000. Generation of strongly asymmetric wave in random seaway. Proc of the 10th International Offshore and Polar Engineering Conference, Seattle.

[31] Kim, N.S., Kim, C.H., 2003. Simulation of Draupner freak wave impact force on a vertical truncated cylinder. Int J of Offshore and Polar Engineering. 13(4), 260-265.

[32] Bennett, S.S., Hudson, D.A., Temarel, P., 2012. A comparison of abnormal wave generation techniques for experimental modelling of abnormal wave-vessel interactions. Ocean engineering. 51, 34-48. https://doi.org/10.1016/j.oceaneng.2012.05.007.

[33] Wheeler, J.D., 1970. Method for calculating forces produced by irregular waves. J of Petroleum Technology. 249, 359-367. https://doi.org/10.2118/2712-PA.

[34] Kim, C.H., Xu, Y., Zou, J., 1997. Impact and nonimpact on vertical truncated cylinder due to strong and weak asymmetric wave. Int J of Offshore and Polar Engineering. 7(3), 161-167.

[35] Funke, E.R., Mansard, E.P.D., 1982. The control of wave asymmetries in random waves. Proc 18th International Conference on Coastal Engineering. Cape Town, South Africa. https://doi.org/10.1061/9780872623736.046.

[36] Westerweel, J., 1993. Digital particle image velocimetry-Theory and application [dissertation]. Delft: Delft University.

Submitted: $\quad$ 14.01.2017. Hae Jin Choi

Department of offshore business, Samsung Engineering Co. Ltd., Seoul,

Accepted: $\quad$ 19.09.2017. Republic of Korea

Seung Jae Lee, Hyo Jae Jo

Department of Naval Architecture and Ocean Systems Engineering, Korea

Maritime and Ocean University, Busan, Republic of Korea

Gang Nam Lee, Kwang Hyo Jung, kjung@ pusan.ac.kr

Department of Naval Architecture and Ocean Engineering, Pusan National

University, Busan, Republic of Korea 